\title{
Orientation Relationships between Gamma (f.c.c.) and Sigma Phases in an Iron-Chromium-Nickel Alloy*
}

\author{
By Soji Nenno**, Masayoshi Tagaya** and Zenji Nishiyama***
}

\begin{abstract}
Single crystals of an $\mathrm{Fe}-25 \% \mathrm{Cr}-20 \% \mathrm{Ni}$ alloy as grown were of gamma phase (f. c. c. structure). When they were annealed for about 200 hours at $815^{\circ} \mathrm{C}$, a considerable amount of sigma phase precipitated in the f. c. c. matrix. By means of $\mathrm{X}$-ray diffraction the ozientation relationships between gamma and sigma phases were determined to be as follows :

$\gamma(111)\|\sigma(001), \quad \gamma[\overline{1} 10]\| \sigma[110] \ldots \ldots \ldots$ A

$\gamma(111)\|\sigma(001), \quad \gamma[011]\| \sigma[140] \ldots \ldots \ldots \mathrm{B}$

The difference between these two orientation relationships $A$ and $B$ is less than $1^{\circ}$. Based upon the orientation relationships established here, the process of the sigma formation from the gamma matrix was discussed. It is suggested that the nucleation of the sigma phase will occur easier at lattice defects such as stacking faults or twins.
\end{abstract}

(Received May 23, 1962)

\section{Introduction}

The sigma phase has been found to form in a number of binary ${ }^{(1)(2)(3)}$ and ternary ${ }^{(4)}$ alloy systems consisting of transition metal elements, namely, Mn$\mathrm{V},-\mathrm{Cr},-\mathrm{Mo} ; \mathrm{Fe}-\mathrm{V},-\mathrm{Nb},-\mathrm{Cr},-\mathrm{Mo},-\mathrm{W}$; Co-V, -Cr, -Mo, -W ; Ru-Cr, -Mo; Rh-Nb, -Ta ; Pd-Nb; Re-Nb, -Ta, -Cr, -Mo, -W ; Os-Nb, -Ta, -Cr, -Mo, -W ; Ir-Nb, -Ta, -Mo, -W ; Pt-Nb, -Ta for binary systems and Fe-Cr$\mathrm{Ni}$, Fe-Cr-Mo, Cr-Co-Fe, Cr-Co-Ni, Fe-Cr-W, Cr-Co-Mo, $\mathrm{Cr}-\mathrm{Ni}-\mathrm{Mo}, \mathrm{Fe}-\mathrm{Cr}-\mathrm{V}$ for ternary systems. It is well known that the sigma phase is an intermediate phase of electron compound type ${ }^{(1 \sim 4)}$.

The crystal structure of the sigma phase in some alloys such as $\mathrm{Fe}-\mathrm{Cr}^{(5)(6)}, \mathrm{Fe}-\mathrm{Mo}^{(5)}, \mathrm{Co}^{\left(5-r^{(6)}(7)\right.}$, and $\mathrm{Ni}-\mathrm{V}^{(8)}$ has already been determined.

The space group is $\mathrm{P} 4 / \mathrm{mnm}$. In the case of $\mathrm{Fe}$ $\mathrm{Cr}^{(5)}$, for example, the tetragonal unit cell $\left(a_{0}=\right.$ $8.800 \AA, c_{0}=4.544 \AA$ ) contains 30 atoms. Fig. 1 shows a projection on (001) plane of atomic arrangements of four unit cells of sigma structure in which a quasihexagonal network of atoms indicated by solid circles and that indicated by open circles are separated by $c_{0} / 2$ from each other. Atoms indicated by double circles are not in these planes, but situated at $c_{0} / 4$ and $3 c_{0} / 4$. Most of the sigma forming elements are of b.c.c., f.c.c., or h.c.p. structure and it is generally

**** Institute of Scientific and Industrial Research, Osaka University, Sakai, Osaka.

** Department of Metallurgy, Faculty of Engineering, Osaka Univeraity, Osaka.

* This paper was presented at the 1961 Spring meeting of The Japan Institute of Metals, Tokyo, Japan.

(1) A. H. Sully : J. Inst. Metals, 80 (1951), 173.

(2) P. Greenfield and P. A. Beck : Trans. AIME, 206 (1956), 265.

(3) A. G. Knapton : J. Inst. Metals, 87 (1958), 28.

(4) S. Rideout, W. D. Manly, E. L. Kamen, B. S. Lement and P. A. Beck: Trans. AIME, 191 (1951), 872.

(5) B. G. Bergman and D. P. Shomaker: J. Chem. Phys., 19 (1951), 515 ; Acta Cryst., 7 (1954), 857.

(6) G. J. Dickins, A. M. B. Douglas and W. H. Taylor: J. Iron Steel Inst., 167 (1951), 27.

(7) J.S. Kasper, B. F. Decker and J. R. Belanger: J. Appl. Phys., 22 (1951), 361.

(8) W. B. Pearson and J. W. Christian: Acta Cryst., 5(1952), 157. known that in many alloy systems the sigma phase forms from one of these structures. It is of interest to know the mechanism of the sigma formation from

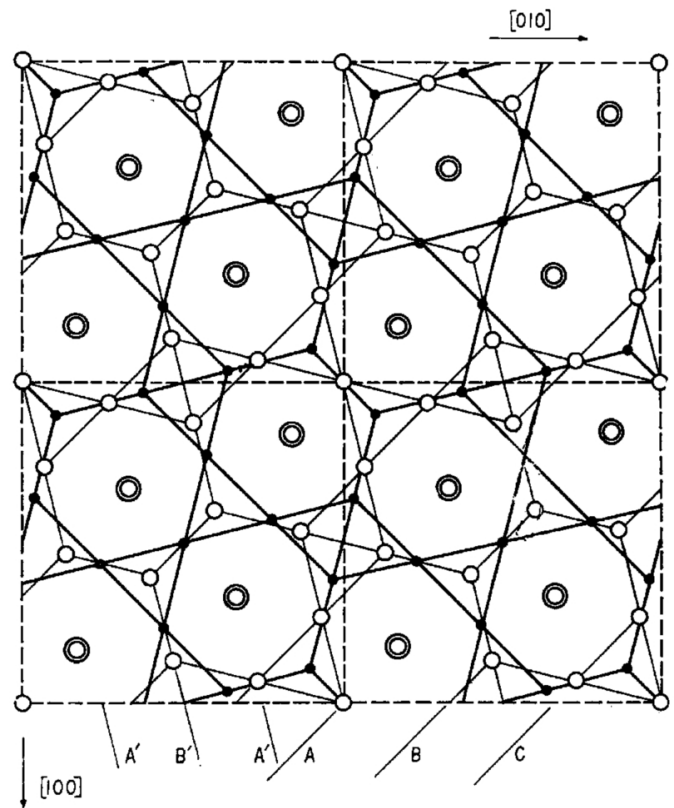

Fig. 1 A projection of the sigma phase structure on the (001) plane (four unit cells put together).

such structures. The first step for this will be to determine the crystallographic relationships between them.

In the present investigation the orientation relationships between sigma and f.c.c. structures have been determined by means of $\mathrm{X}$-ray diffraction ${ }^{(9)}$ and on the basis of these orientation relationships some considerations were given to the mechanism of the sigma formation from the f.c.c. matrix. The alloy used is of the AISI 310 type ( $25 \%$ of $\mathrm{Cr}, 20 \%$ of Ni, nearly $1 \%$ of $\mathrm{Si}$, iron the remainder) which has the following characteristics:

(1) It is f.c.c. even when it is furnace-cooled from a high temperature where solution treatment is made,

(9) Z. Nishiyama: Sci. Rep. Tohoku IMP. Univ., 23 (1934), 637. 
for example, $1350^{\circ} \mathrm{C}$.

(2) The sigma phase precipitates on certain crystallographic planes within the f.c.c. matrix as well as at grain boundaries when the alloy is held at a temperature between $650^{\circ}$ and $900^{\circ} \mathrm{C}$. ${ }^{(10)}$

(3) This alloy is widely used as a heat-resisting :steel and the sigma embrittlement in this alloy is of great technical importance. ${ }^{(10)}$

\section{Experimental Procedure}

\section{Preparation of specimens}

Materials used were electrolytic nickel, electrolytic iron, metallic chromium, and metallic silicon. Proper portions of them totalling 800 grams were melted in a Tammann furnace and were cast into a mould $15 \times$ $15 \mathrm{~mm}^{2}$ in cross-section. After homogenizing the ingot, it was forged and rolled to plates $28 \times 2 \mathrm{~mm}^{2}$ in cross-section. These plates were cut into pieces $80 \mathrm{~mm}$ long. The chemical composition of this alloy (in wt \%) was :

C : 0.185 , Si : $0.88, \mathrm{Cr}: 23.39, \mathrm{Ni}: 21.39$,

$\mathrm{Fe}$ : balance.

'To prevent the carbide formation in the alloy, the specimens were decarburized ${ }^{(11)}$ in wet hydrogen for

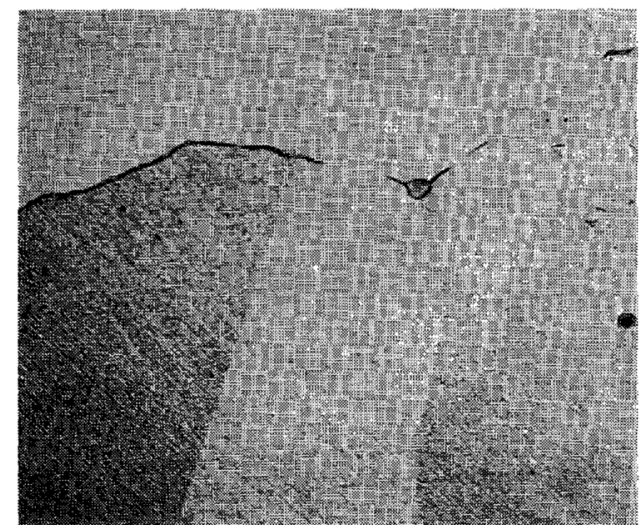

(a)

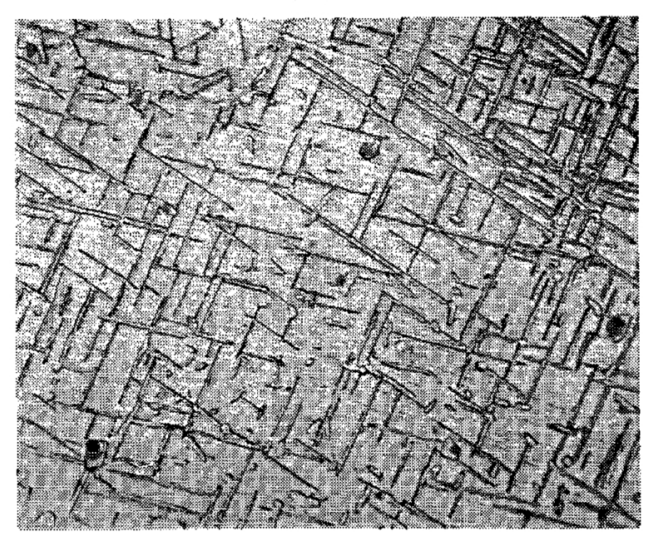

(c) grains $/ \mathrm{mm}^{2}$; strain : at least $8 \%$ in tension : annealing : $1350^{\circ} \mathrm{C}, 5 \mathrm{hr}$. in dry hydrogen).

Single crystals for $\mathrm{X}$-ray work were cut chemically from the coarse-grained specimens. For the microscopic examination and Debye-Scherrer method, polycrystalline specimens were taken from the decarburized plates.

\section{Heat treatment}

The single crystals and the polycrystalline specimens mentioned above were vacuum annealed for various lengths of time up to about $200 \mathrm{hrs}$. at $815^{\circ} \mathrm{C}$, where the sigma phase easily forms. ${ }^{(12)}$ Some of the photomicrographs taken of polycrystalline specimens annealed at $815^{\circ} \mathrm{C}$ are shown in Photo. 1 , in which only the original gamma phase is seen in (a), while in (b), (c) and (d) the sigma phase precipitates are found on several crystallographic planes in the matrix and along the grain boundaries, and the amount of the precipitates increases with annealing time in the order (b), (c) and (d).

\section{Measurement of the lattice constant}

The lattice constant of the f.c.c. matrix of the specimen annealed for about 200 hrs., as obtained by

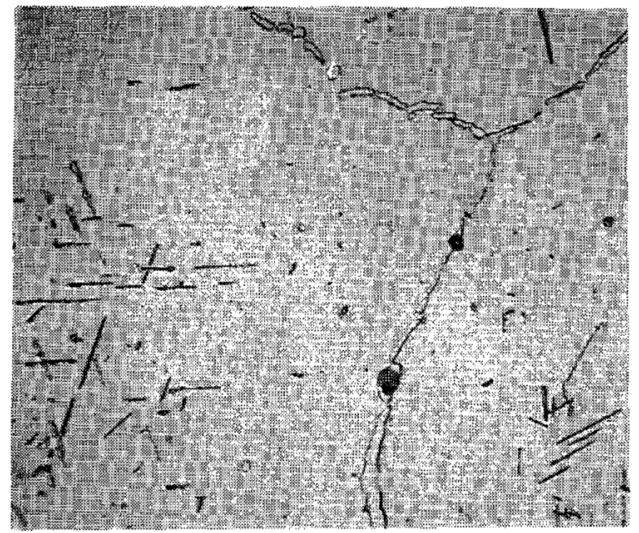

(b)

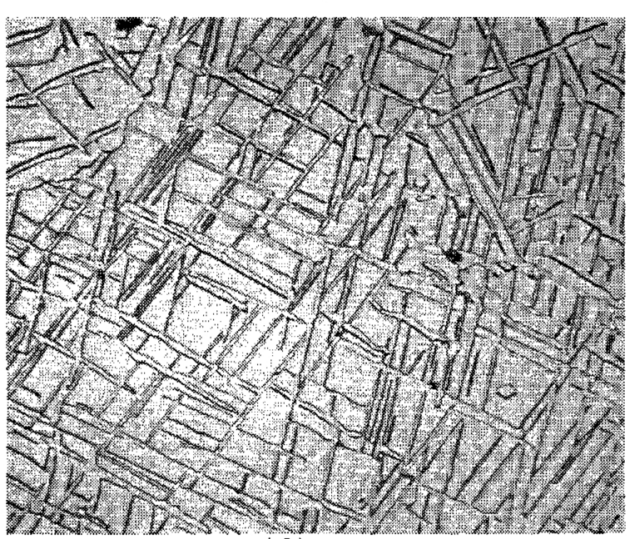

(d)

Photo. 1 Photomicrographs showing the sigma phase precipitation from the gamma matrix. (Marble etch, $\times 400$ )

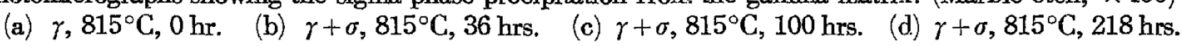

t68 hours at $1050^{\circ} \mathrm{C}$. The final carbon content was found to be less than $0.01 \%$. Fairly coarse grained $\left(5 \times 25 \mathrm{~mm}^{2}\right)$ specimens were obtained by applying the strain-annealing method ${ }^{(11)}$ (initial grain size : 16

(10) J. I. Morley and H. W. Kirkby : J. Iron Steel Inst., 172 (1952), 129. the use of a back reflection focusing camera, was found to be $3.5871 \AA$ which is in good agreement with that obtained by Rees et al., ${ }^{(13)}$ namely $a_{0}=3.5874 \AA$.

(11) M. Yamamoto and R. Miyasawa: J. Japan Inst. Metals, 16 (1952), 300 .

(12) G. J. Guarnieri, J. Miller and F. J. Vawter: Trans. ASM, 42 (1950), 981. 
In order to find the lattice constants of the sigma phase precipitated, it was necessary to isolate the sigma precipitate, because the intensity of the reflections from the sigma phase was very weak as compared to those from the f.c.c. matrix. The sigma precipitate was extracted electrolytically(14) from a specimen annealed for about $200 \mathrm{hrs}$. at $815^{\circ} \mathrm{C}$. Using this extracted residue, the precipitate was identified as the sigma phase and the lattice constants were determined as $a_{0}=8.779 \AA, c_{0}=4.533 \AA$ and $a_{0} / c_{0}=1.937$, using a $114.6 \mathrm{~mm}$ diameter DebyeScherrer camera with $\operatorname{Cr}-K \alpha$ radiation $\left(\lambda_{K_{\alpha 1}}=2.28962\right.$ $\AA, \lambda_{K_{\alpha 2}}=2.29351 \AA$ ).

\section{X-ray single crystal work}

The specimen, in which sigma crystallites were precipitated by annealing an original gamma single crystal for about $200 \mathrm{hrs}$. at $815^{\circ} \mathrm{C}$, was mounted on the goniometer head of a $70 \mathrm{~mm}$ diameter rotating crystal camera. A number of rotation and oscillation photographs about $\gamma[001]$ and $\gamma[0 \overline{\mathrm{I}} 1]$ axes were taken with $\mathrm{Mo}-K \alpha$ or $\mathrm{Cr}-K \alpha$ radiations. A cylindrical film cassette and a flat cassette were used in the cases of $\mathrm{Cr}$ and Mo radiations, respectively. When the specimen is rotated to a position where reflection may occur, the position $\delta$ of the interference spot is given by $\cos \delta=\frac{\cos \rho}{\cos \theta} \cdots . .(1)$, where $\rho$ denotes the angle between the z-axis and the normal of a reflecting plane and $\theta$ is the Bragg angle. In case the flat film cassette is used, the position $\delta$ of the interference spot can be directly measured as illustrated in Fig. 2, $\mathrm{n}$ which the $\mathrm{z}$-axis is taken as the axis of rotation and

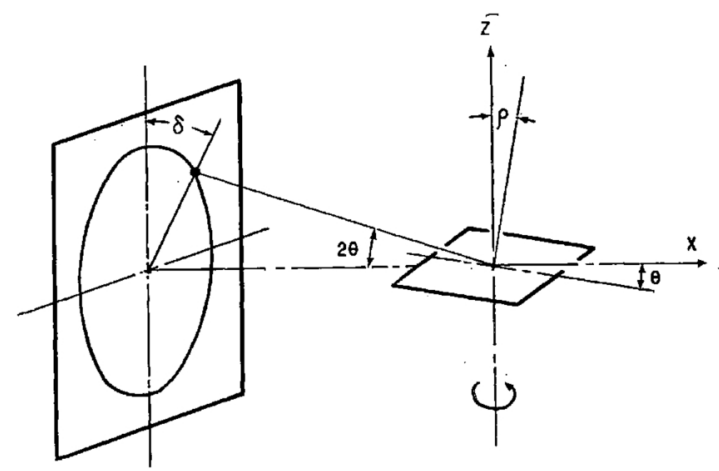

Fig. 2 Diagram of the position of a reflection spot from the crystal referred to the incident $X$-ray beams.

the $\mathrm{x}$-axis as the direction to $\mathrm{X}$-ray source. In case the cylindrical film cassette is used, the value of $\delta$, as defined above, corresponding to any interference spot appearing on a cylindrical film can be easily determined by the use of a Bernal chart appropriate to the $70 \mathrm{~mm}$ diameter rotating crystal camera, using the following equation together with equation (1) :

$$
\tan \rho=\xi / \zeta,
$$

where $\xi$ is the perpendicular distance from a reciprocal lattice point to the axis of rotation; and $\zeta$ is the

(13) W. P. Rees, B. D. Burns and A. J. Cook: J. Iron Steel Inst., 162 (1949), 325.

(14) R. E. Lismer, L. Pryce and K. W. Andrews : J. Iron Steel Inst., 171 (1952), 49. distance parallel to the axis from the origin to the point.

\section{Rotation and Oscillation Photographs}

Photos. 2 and 3 are the rotation photographs about. the $\gamma[001]$ and $\gamma[0 \overline{1} 1]$ axes, respectively, taken with $\mathrm{Cr}-K \alpha$ radiation. In these photographs the inter-

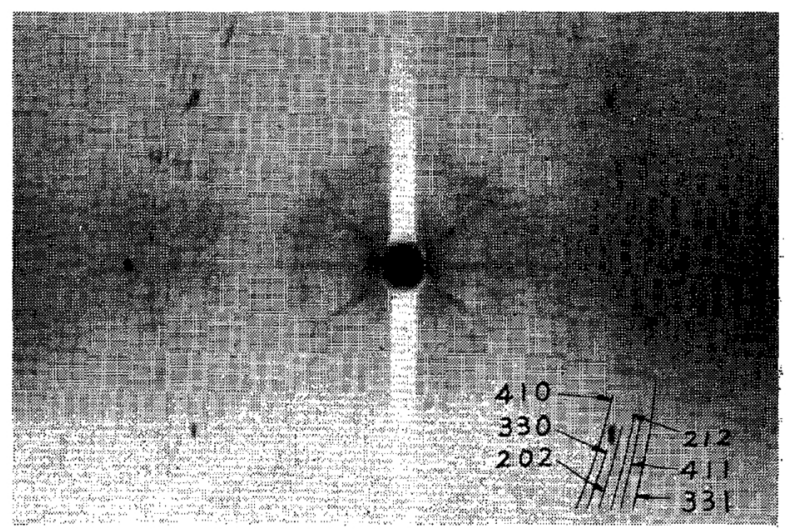

Photo. 2 Rotation photograph about the $r$ [001] axis, $\mathrm{Cr}-K \alpha$ radiation. Specimen : $815^{\circ} \mathrm{C}, 218 \mathrm{hrs}$; camera diameter $70 \mathrm{~mm}$.

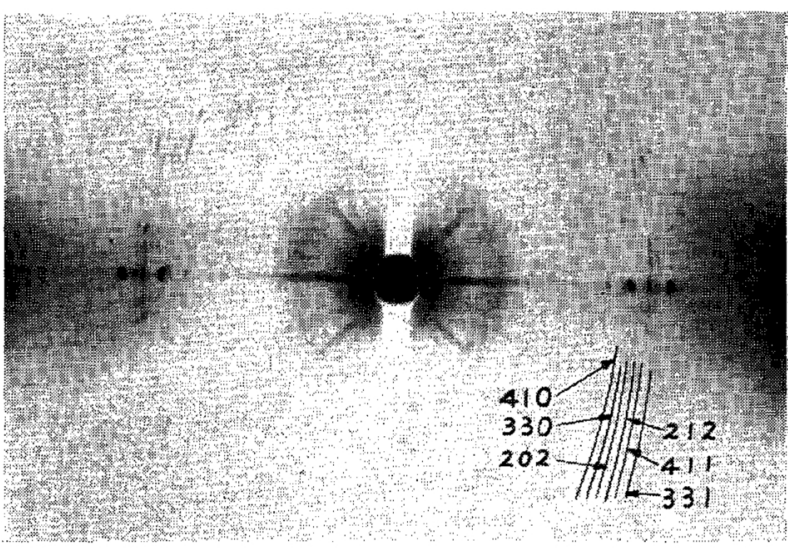

Photo. 3 Rotatation photogxagh about the $\gamma$ [011] axis, $\mathrm{Cr}-K \alpha$ radiation. Specimen : $815^{\circ} \mathrm{C}, 218 \mathrm{hrs}$. camera diameter $70 \mathrm{~mm}$.

ference spots from the gamma crystal and sigma crystallites can be seen. The values of position $\delta$ of the interference spot, as obtained from each of these photographs by the use of relations (1) and (2) arelisted in Table 1 for $\gamma[0 \overline{1} 1]$-rotation and in Table 2 for $\gamma[001]$-rotation. Photo. 4 is the rotation photograph about $\gamma[0 \overline{1} 1]$ axis taken with Mo- $K \alpha$ radiation in order to observe the sigma spots with small $\rho$ values ( $\delta$ is less than about $30^{\circ}$ in this case). Photo. 5 (a), (b), (c) and (d) are the oscillation photographs about $\gamma[001]$ axis with about $2^{\circ}$ of oscillation at various angles of incidence with respect to $\gamma[\overline{100}]$ direction. Photo. $6(\mathrm{a}),(\mathrm{b}), \cdots(\mathrm{h})$ are those about $\gamma[0 \overline{1} 1]$ axis.

\section{Possible Orientation Relationships and Comparison of Atomic Arrangements in Gamma and Sigma Lattices}

When a new phase is formed in a matrix the factors. which affect the orientation relationship between old and new crystal lattices may be the following : (1) The atomic arrangements of both crystals at or near the interface are in register or similar to each other-the 
energy required to form the interface is to be small. cases, the most densely packed atomic planes seem to (2) In case where the new crystal is formed by simple satisfy conditions, (1) and (2). In a sigma phase lattice slip, the activation energy is to be small. In most structure, the most densely packed atomic planes are

Table 1 Analysis of the rotation photograph about $\gamma[011]$ axis (Photo. 3).

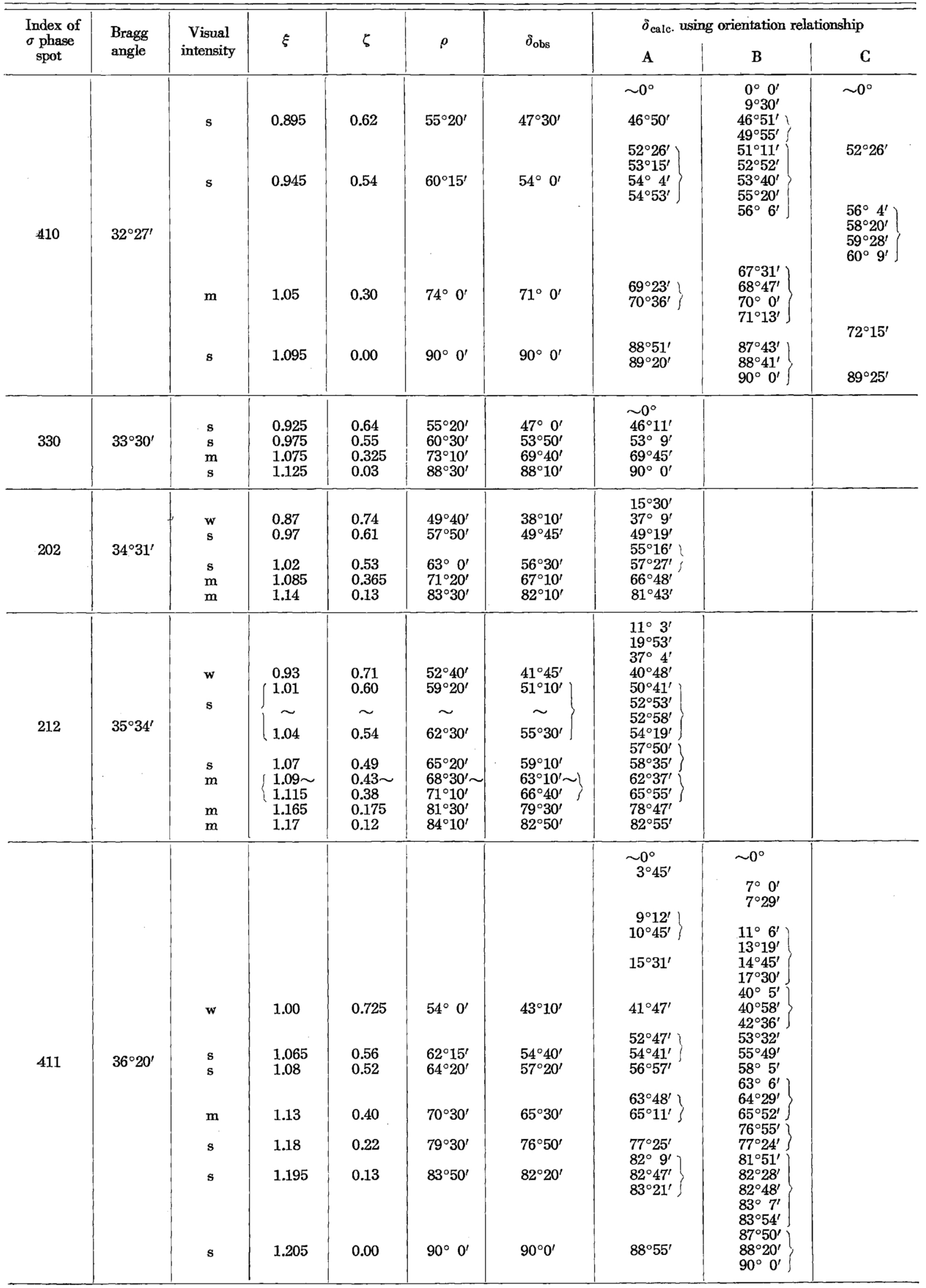


Table 1 (continued)

\begin{tabular}{|c|c|c|c|c|c|c|c|c|c|}
\hline \multirow{2}{*}{$\begin{array}{c}\text { Index of } \\
\sigma \text { phase } \\
\text { spot }\end{array}$} & \multirow{2}{*}{$\begin{array}{l}\text { Bragg } \\
\text { angle }\end{array}$} & \multirow{2}{*}{$\begin{array}{l}\text { Visual } \\
\text { intensity }\end{array}$} & \multirow{2}{*}{$\xi$} & \multirow{2}{*}{$\zeta$} & \multirow[b]{2}{*}{$\rho$} & \multirow{2}{*}{$\delta_{\text {obs. }}$} & \multicolumn{3}{|c|}{$\delta_{\text {calc. }}$ using orientation relationship } \\
\hline & & & & & & & A & B & C \\
\hline 331 & $37^{\circ} 20^{\prime}$ & $\begin{array}{c}\mathbf{w} \\
\mathbf{s} \\
\mathbf{w} \\
\mathbf{s} \\
\mathrm{s} \\
\mathrm{m} \\
\mathrm{s}\end{array}$ & $\begin{array}{l}0.98 \\
1.10 \\
1.16 \\
1.21 \\
1.225 \\
1.23 \\
1.23\end{array}$ & $\begin{array}{l}0.73 \\
0.55 \\
0.40 \\
0.23 \\
0.145 \\
0.09 \\
0.00\end{array}$ & $\begin{array}{l}53^{\circ} 20^{\prime} \\
63^{\circ} 30^{\prime} \\
71^{\circ} 0^{\prime} \\
79^{\circ} 15^{\prime} \\
83^{\circ} 15^{\prime} \\
85^{\circ} 50^{\prime} \\
90^{\circ} 0^{\prime}\end{array}$ & $\begin{array}{l}41^{\circ} 20^{\prime} \\
55^{\circ} 45^{\prime} \\
65^{\circ} 50^{\prime} \\
76^{\circ} 25^{\prime} \\
81^{\circ} 30^{\prime} \\
84^{\circ} 45^{\prime} \\
90^{\circ} 0^{\prime}\end{array}$ & $\begin{array}{r}3^{\circ} 13^{\prime} \\
7^{\circ} 41^{\prime} \\
40^{\circ} 50^{\prime} \\
55^{\circ} 6^{\prime} \\
64^{\circ} 46^{\prime} \\
76^{\circ} 26^{\prime} \\
81^{\circ} 37^{\prime} \\
84^{\circ} 30^{\prime} \\
90^{\circ} 0^{\prime}\end{array}$ & & \\
\hline
\end{tabular}

Table 2 Analysis of the rotation photograph about $\gamma$ [001] axis (Photo. 2).

\begin{tabular}{|c|c|c|c|c|c|c|c|}
\hline $\begin{array}{l}\text { Index of } \\
\sigma \text { phase } \\
\text { spot }\end{array}$ & $\begin{array}{c}\text { Bragg } \\
\text { angle }\end{array}$ & $\begin{array}{c}\text { Visual } \\
\text { intensity }\end{array}$ & $\xi$ & $\zeta$ & $\rho$ & $\delta_{\text {obs. }}$ & $\begin{array}{c}\delta_{\text {calc. }} \text { using orientation } \\
\text { relationship } \\
\mathbf{A}\end{array}$ \\
\hline 410 & $32^{\circ} 27^{\prime}$ & $\begin{array}{c}\mathbf{s} \\
\mathbf{s} \\
\mathbf{m}\end{array}$ & $\begin{array}{l}0.775 \\
1.00 \\
1.10\end{array}$ & $\begin{array}{l}0.77 \\
0.44 \\
0.00\end{array}$ & $\begin{array}{l}45^{\circ} 20^{\prime} \\
66^{\circ} 10^{\prime} \\
90^{\circ} 0^{\prime}\end{array}$ & $\begin{array}{l}33^{\circ} 30^{\prime} \\
61^{\circ} 20^{\prime} \\
90^{\circ} 0^{\prime}\end{array}$ & $\left.\begin{array}{l}14^{\circ} 38^{\prime} \\
32^{\circ} 13^{\prime} \\
33^{\circ} 56^{\prime} \\
60^{\circ} 9^{\prime} \\
61^{\circ} 59^{\prime} \\
89^{\circ} 4^{\prime}\end{array}\right\}$ \\
\hline 330 & $33^{\circ} 30^{\prime}$ & $\begin{array}{l}\mathbf{s} \\
\mathbf{s} \\
\mathbf{w}\end{array}$ & $\begin{array}{l}0.80 \\
1.03 \\
1.13\end{array}$ & $\begin{array}{l}0.79 \\
0.45 \\
0.00\end{array}$ & $\begin{array}{l}45^{\circ} 20^{\prime} \\
66^{\circ} 20^{\prime} \\
90^{\circ} 0^{\prime}\end{array}$ & $\begin{array}{l}32^{\circ} 30^{\prime} \\
61^{\circ} 10^{\prime} \\
90^{\circ} 0^{\prime}\end{array}$ & $\begin{array}{l}11^{\circ} 14^{\prime} \\
32^{\circ} 1^{\prime} \\
60^{\circ} 41^{\prime} \\
90^{\circ} 0^{\prime}\end{array}$ \\
\hline 202 & $34^{\circ} 31^{\prime}$ & $\begin{array}{l}\mathrm{m} \\
\mathrm{m} \\
\mathrm{w} \\
\mathrm{w}\end{array}$ & $\begin{array}{l}0.92 \\
1.05 \\
1.11 \\
1.14\end{array}$ & $\begin{array}{l}0.68 \\
0.47 \\
0.275 \\
0.16\end{array}$ & $\begin{array}{l}53^{\circ} 30^{\prime} \\
65^{\circ} 50^{\prime} \\
76^{\circ} 10^{\prime} \\
82^{\circ} 0^{\prime}\end{array}$ & $\begin{array}{l}43^{\circ} 50^{\prime} \\
60^{\circ} 10^{\prime} \\
73^{\circ} 10^{\prime} \\
80^{\circ} 20^{\prime}\end{array}$ & $\begin{array}{l}19^{\circ} 14^{\prime} \\
42^{\circ} 15^{\prime} \\
59^{\circ} 41^{\prime} \\
72^{\circ} 29^{\prime} \\
79^{\circ} 27^{\prime}\end{array}$ \\
\hline 212 & $35^{\circ} 34^{\prime}$ & $\begin{array}{l}\mathbf{m} \\
\mathrm{m} \\
\mathbf{w} \\
\mathbf{w} \\
\mathbf{w} \\
\mathrm{w} \\
\mathrm{w}\end{array}$ & $\begin{array}{l}0.92 \\
0.96 \\
1.07 \\
1.10 \\
1.15_{5} \\
1.16 \\
\\
1.17_{5}\end{array}$ & $\begin{array}{l}0.73 \\
0.67 \\
0.48 \\
0.425 \\
0.26 \\
0.23 \\
\\
0.12\end{array}$ & $\begin{array}{l}51^{\circ} 30^{\prime} \\
55^{\circ} 0^{\prime} \\
65^{\circ} 50^{\prime} \\
68^{\circ} 50^{\prime} \\
77^{\circ} 20^{\prime} \\
78^{\circ} 50^{\prime} \\
84^{\circ} 10^{\prime}\end{array}$ & $\begin{array}{l}40^{\circ} 0^{\prime} \\
45^{\circ} 10^{\prime} \\
59^{\circ} 50^{\prime} \\
63^{\circ} 40^{\prime} \\
74^{\circ} 20^{\prime} \\
76^{\circ} 10^{\prime} \\
82^{\circ} 50^{\prime}\end{array}$ & $\left.\begin{array}{r}8^{\circ} 1^{\prime} \\
18^{\circ} 37^{\prime} \\
39^{\circ} 21^{\prime} \\
44^{\circ} 20^{\prime} \\
59^{\circ} 4^{\prime} \\
62^{\circ} 53^{\prime} \\
73^{\circ} 39^{\prime} \\
76^{\circ} 11^{\prime} \\
82^{\circ} 3^{\prime} \\
82^{\circ} 57^{\prime}\end{array}\right\}$ \\
\hline 411 & $36^{\circ} 20^{\prime}$ & $\begin{array}{l}\mathrm{m} \\
\mathrm{w} \\
\mathrm{s} \\
\mathrm{s} \\
\mathrm{s}\end{array}$ & $\begin{array}{l}0.95 \\
1.05 \\
1.11 \\
1.17 \\
1.20\end{array}$ & $\begin{array}{l}0.725 \\
0.58 \\
0.47 \\
0.29 \\
0.15\end{array}$ & $\begin{array}{l}52^{\circ} 40^{\prime} \\
61^{\circ} 0^{\prime} \\
67^{\circ} 0^{\prime} \\
76^{\circ} 0^{\prime} \\
82^{\circ} 50^{\prime}\end{array}$ & $\begin{array}{l}41^{\circ} 15^{\prime} \\
53^{\circ} 0^{\prime} \\
61^{\circ} 0^{\prime} \\
72^{\circ} 30^{\prime} \\
81^{\circ} 5^{\prime}\end{array}$ & $\left.\begin{array}{l}39^{\circ} 3^{\prime} \\
41^{\circ} 25^{\prime} \\
52^{\circ} 12^{\prime} \\
60^{\circ} 9^{\prime} \\
61^{\circ} 10^{\prime} \\
71^{\circ} 20^{\prime} \\
73^{\circ} 12^{\prime} \\
80^{\circ} 21^{\prime} \\
81^{\circ} 54^{\prime}\end{array}\right\}$ \\
\hline 331 & $37^{\circ} 20^{\prime}$ & $\begin{array}{c}\mathrm{m} \\
\mathrm{w} \\
\mathrm{s} \\
\mathrm{m} \\
\mathrm{s}\end{array}$ & $\begin{array}{l}0.98 \\
1.06 \\
1.13 \\
1.20 \\
1.23\end{array}$ & $\begin{array}{l}0.73 \\
0.62 \\
0.49 \\
0.29 \\
0.16\end{array}$ & $\begin{array}{l}53^{\circ} 20^{\prime} \\
59^{\circ} 40^{\prime} \\
66^{\circ} 40^{\prime} \\
76^{\circ} 30^{\prime} \\
82^{\circ} 40^{\prime}\end{array}$ & $\begin{array}{l}41^{\circ} 20^{\prime} \\
50^{\circ} 30^{\prime} \\
60^{\circ} 10^{\prime} \\
73^{\circ} 0^{\prime} \\
80^{\circ} 40^{\prime}\end{array}$ & $\begin{array}{l}39^{\circ} 46^{\prime} \\
50^{\circ} 44^{\prime} \\
59^{\circ} 29^{\prime} \\
72^{\circ} 27^{\prime} \\
80^{\circ} 27^{\prime}\end{array}$ \\
\hline
\end{tabular}

$\langle 001),\{140\}$ and $\{110\}$, while those of the gamma phase (f.c.c) are $\{111\}$. Let us compare the atomic arrangements in these planes.

\section{1. $\sigma(001)$ and $\gamma(\mathbf{1 1 1})$}

As shown in Fig. 3, atoms in the $\gamma$ (111) plane form a triangular network, while those in the $\sigma(001)$ plane do the same except the atoms at the centers of pseudo. hexagonal nets which are shifted in the c-direction by $c_{0} / 4$. The edge length of an elementary triangle ADF in Fig. 3 (a) is $2.5365 \AA$, while each edge length of an elementary triangle $\mathrm{OAB}$ in Fig. $3(\mathrm{~b})$ is given as follows :

$$
A B=\sqrt{2} a_{0} / 5=2.483 \AA
$$

$O A=O B=\sqrt{(A B / 2)^{2}+\left(\sqrt{\left.2 a_{0} / 6\right)^{2}}=2.412 \AA\right.}$,

Miller index of direction $\mathrm{OA}:[410]$.

Thus $\gamma(111)$ and $\sigma(001)$ planes are very similar to each other with respect to the atomic arrangements. and distances. In superposing triangle $\mathrm{OAB}$ upon triangle ADF, there may be two ways as shown in Fig. 4 (a) and (b), which lead to two slightly different orientation relationships :

$$
\left.\begin{array}{l}
\gamma(111) \| \sigma(001) \\
\gamma[\overline{110}] \| \sigma[\overline{1} 10],
\end{array}\right\}
$$

and

$$
\left.\begin{array}{l}
\gamma(111) \| \sigma(001) \\
\gamma[01 \bar{I}] \| \sigma[140] .
\end{array}\right\}
$$


For orientation relationship $A$, the relation between the axes $x, y, z$ of gamma and the axes $x^{\prime}, y^{\prime}, z^{\prime}$ of sigma can be expressed by the following matrix of

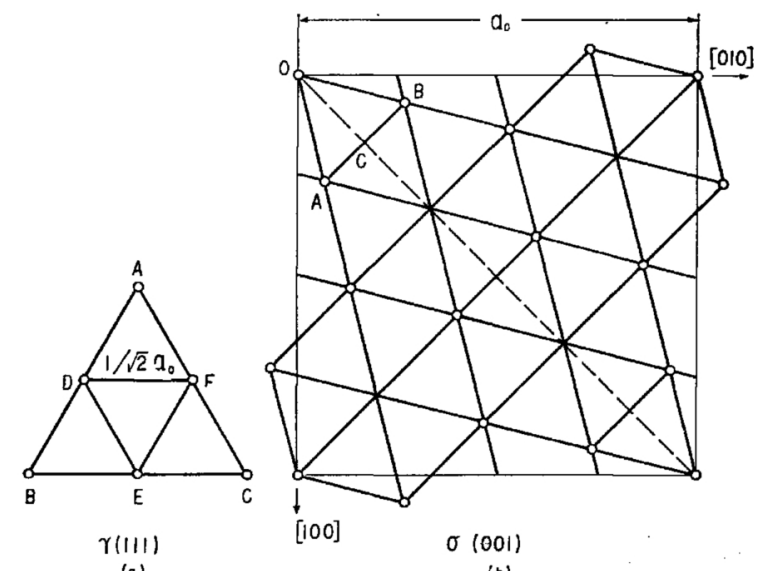

(b)

Fig. 3 (a) A (111) plane of gamma phase structure.

(b) A basal plane (001) of sigma phase structure.

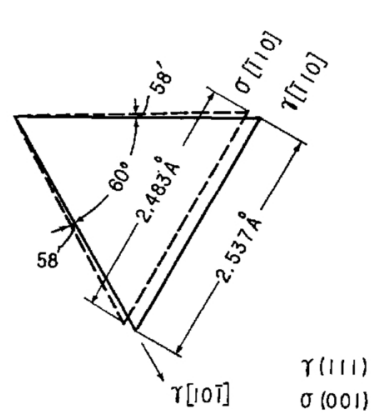

(a)

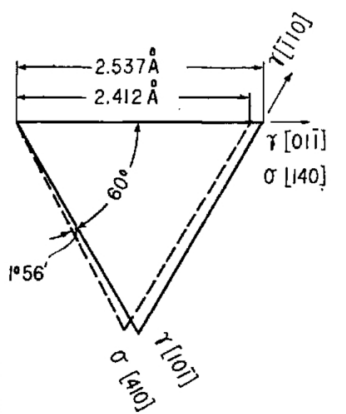

(b)
Fig. 4 Illustration of two orientation relationships:

(a) orientation relationship $A$,

(b) orientation relationship $B$.

linear transformation :

\begin{tabular}{|c|c|c|c|}
\hline \multirow{2}{*}{$\begin{array}{l}\text { Principal } \\
\text { axes }\end{array}$} & \multicolumn{3}{|c|}{$\sigma$ Crystal } \\
\hline & $\mathbf{x}^{\prime}$ & $y^{\prime}$ & $\mathbf{z}^{\prime}$ \\
\hline $\mathbf{x}$ & $\frac{1}{2}+\frac{1}{\sqrt{12}}$ & $-\frac{1}{2}+\frac{1}{\sqrt{12}}$ & $\frac{1}{\sqrt{3}}$ \\
\hline$\gamma$ Crystal y & $-\frac{1}{2}+\frac{1}{\sqrt{12}}$ & $\frac{1}{2}+\frac{1}{\sqrt{12}}$ & $\frac{1}{\sqrt{3}}$ \\
\hline Z & $-\frac{1}{\sqrt{3}}$ & $-\frac{1}{\sqrt{3}}$ & $\frac{1}{\sqrt{3}}$ \\
\hline
\end{tabular}

The number of possible transformations (variants) is 12 , and they are classified by parallelism relations

Table 3 Classification of twelve orientations of $\sigma$ crystals.

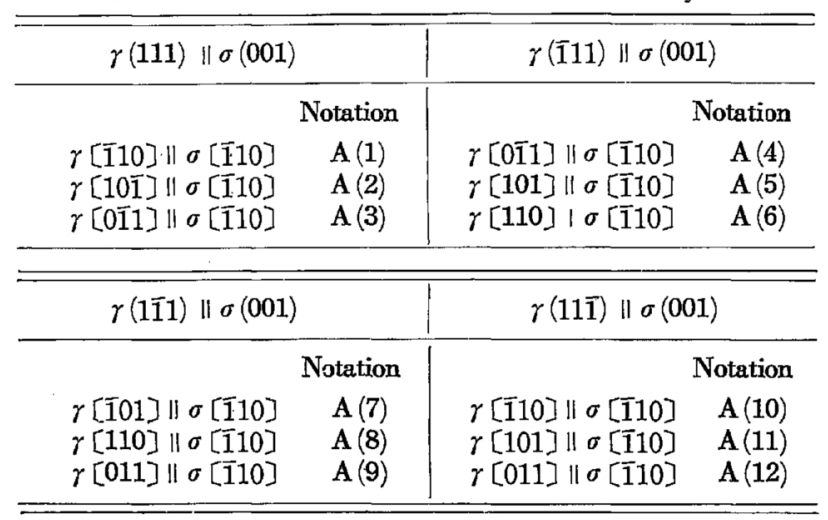

and are given in Table 3 . Using this relation, the direction cosines of the planes $\{140\},\{330\},\{202\}$, $\{212\},\{411\}$ and $\{331\}$ of sigma crystal referred to gamma principal axes are calculated as given in Table 4.

For orientation relationship B, the transformation matrix is :

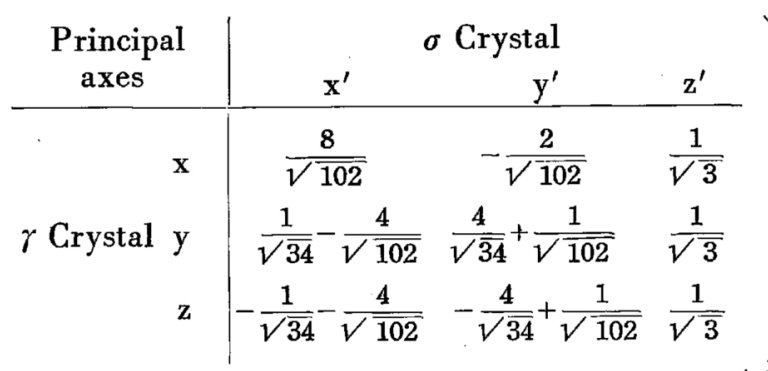

The number of variants is 24 . In the same way as before, the direction cosines of the net planes of a sigma crystal are calculated. In Table 5, only those vales for the planes $\{140\}$ and $\{411\}$ are listed.

\section{2. $\sigma(\mathbf{1 1 0})$ and $\gamma(\mathbf{1 1 1})$}

Let us look at a $\sigma(110)$ plane in Fig. 1, say plane A. Some atoms lie in this plane, and others do not but deviate from this plane by various amounts up to half the spacing $(2.068 \AA)$ of $\sigma(330)$ plane. If we assume that the atoms in the latter category lay in the $\sigma(110)$ plane, all the atoms in this plane would be arranged as a network consisting of a combination of triangles, the dimension of which is nearly the same as that of triangle ADF in $\gamma(111)$ plane shown in Fig. $3(\mathrm{a})$, with two different orientations : namely, one with its edge parallel to $\sigma[\overline{1} 10$, the other with its edge parallel to $\sigma[001]$. Thus, we have the following possible orientation relationships :

$$
\begin{aligned}
& \left.\begin{array}{l}
\sigma(110) \| \gamma(111) \\
\sigma[001] \| \gamma[\overline{112}],
\end{array}\right\} \\
& \text { or } \quad \sigma(110) \| \gamma(111) \\
& \sigma[001] \| \gamma[\overline{1} 10] .\}
\end{aligned}
$$

In the case of orientation relationship $\mathrm{C}$, the spacing $\left(c_{0}=4.533 \AA\right)$ of $\sigma(001)$ corresponds to the length $A E(4.393 \mathrm{~A})$ of triangle $\mathrm{ABC}$ of $\gamma(111)$ in Fig. $3(\mathrm{a})$, while in orientation relationship $D$ the spacing of $\sigma(001)$ corresponds to edge length $B C(5.073 \AA)$ of triangle $\mathrm{ABC}$ of $\gamma(111)$. Since the matching between the atomic arrangements in $\gamma(111)$ and $\sigma(110)$ is better in orientation relationship $\mathrm{C}$, this relationship will only be given further consideration. It is expressed by the following matrix :

$\left.\begin{array}{r|ccc}\begin{array}{c}\text { Principal } \\ \text { axes }\end{array} & \mathrm{x}^{\prime} & \mathrm{y}^{\prime} & \mathrm{z}^{\prime} \\ \hline \mathrm{x} & -\frac{1}{\sqrt{6}}-\frac{1}{2} & \frac{1}{\sqrt{6}}+\frac{1}{2} & -\frac{1}{\sqrt{6}} \\ r \text { Crystal } \mathrm{y} & -\frac{1}{\sqrt{6}}+\frac{1}{2} & -\frac{1}{\sqrt{6}}-\frac{1}{2} & -\frac{1}{\sqrt{6}} \\ \mathrm{z} & -\frac{1}{\sqrt{6}} & -\frac{1}{\sqrt{6}} & \frac{2}{\sqrt{6}}\end{array}\right\}$


Table 4 Direction cosines of $\sigma\{h k l\}$ referred to gamma principal axes, as calculated by using orientation relationship A.

\begin{tabular}{|c|c|c|c|}
\hline$\sigma(h k l)$ & $\cos \alpha$ & $\cos \beta$ & $\cos \gamma$ \\
\hline 410 & $\frac{1}{\sqrt{17}}\left(\frac{3}{2}+\frac{5}{\sqrt{12}}\right)$ & $\frac{1}{\sqrt{17}}\left(-\frac{3}{2}+\frac{5}{\sqrt{12}}\right)$ & $-\frac{5}{\sqrt{51}}$ \\
\hline 140 & $\frac{1}{\sqrt{17}}\left(-\frac{3}{2}+\frac{5}{\sqrt{12}}\right)$ & $\frac{1}{\sqrt{17}}\left(\frac{3}{2}+\frac{5}{\sqrt{12}}\right)$ & $-\frac{5}{\sqrt{51}}$ \\
\hline$\overline{4} 10$ & $\frac{1}{\sqrt{17}}\left(-\frac{5}{2}-\frac{3}{\sqrt{12}}\right)$ & $\frac{1}{\sqrt{17}}\left(\frac{5}{2}-\frac{3}{\sqrt{12}}\right)$ & $\frac{3}{\sqrt{51}}$ \\
\hline$\overline{1} 40$ & $\frac{1}{\sqrt{17}}\left(-\frac{5}{2}+\frac{3}{\sqrt{12}}\right)$ & $\frac{1}{\sqrt{17}}\left(\frac{5}{2}+\frac{3}{\sqrt{12}}\right)$ & $-\frac{3}{\sqrt{51}}$ \\
\hline 330 & $\frac{1}{\sqrt{6}}$ & $\frac{1}{\sqrt{6}}$ & $-\sqrt{\frac{2}{3}}$ \\
\hline$\overline{3} 30$ & $-\frac{1}{\sqrt{2}}$ & $\frac{1}{\sqrt{2}}$ & 0 \\
\hline 202 & {$\left[\frac{1}{2}+\frac{1}{\sqrt{12}}+\frac{1}{\sqrt{3}}(a \mid c)\right] / \sqrt{1+(a \mid c)^{2}}$} & {$\left[-\frac{1}{2}+\frac{1}{\sqrt{12}}+\frac{1}{\sqrt{3}}(a \mid c)\right] / \sqrt{1+(a \mid c)^{2}}$} & {$\left[-\frac{1}{\sqrt{3}}+\frac{1}{\sqrt{3}}(a \mid c)\right] / \sqrt{1+(a \mid c)^{2}}$} \\
\hline 022 & {$\left[-\frac{1}{2}+\frac{1}{\sqrt{12}}+\frac{1}{\sqrt{3}}(a \mid c)\right] / \sqrt{1+(a \mid c)^{2}}$} & {$\left[\frac{1}{2}+\frac{1}{\sqrt{12}}+\frac{1}{\sqrt{3}}(a \mid c)\right] / \sqrt{1+(a \mid c)^{2}}$} & {$\left[-\overline{1} \overline{\bar{\beta}^{3}}+\frac{1}{\sqrt{\overline{3}}}(a \mid c)\right] / \sqrt{1+(a \mid c)^{2}}$} \\
\hline$\overline{2} 02$ & {$\left[-\frac{1}{2}-\frac{1}{\sqrt{12}}+\frac{1}{\sqrt{3}}(a \mid c)\right] / \sqrt{1+(a \mid c)^{2}}$} & {$\left[\frac{1}{2}-\frac{1}{\sqrt{12}}+\frac{1}{\sqrt{3}}(a \mid c)\right] / \sqrt{1+(a \mid c)^{2}}$} & {$\left[\frac{1}{\sqrt{3}}+\frac{1}{\sqrt{3}}(a \mid c)\right] / \sqrt{1+(a \mid c)^{2}}$} \\
\hline $0 \overline{2} 2$ & {$\left[\frac{1}{2}-\frac{1}{\sqrt{12}}+\frac{1}{\sqrt{3}}(a \mid c)\right] / \sqrt{1+(a \mid c)^{2}}$} & {$\left[-\frac{1}{2}-\frac{1}{\sqrt{12}}+\frac{1}{\sqrt{3}}(a \mid c)\right] / \sqrt{1+(a \mid c)^{2}}$} & {$\left[\frac{1}{\sqrt{3}}+\frac{1}{\sqrt{3}}(a \mid c)\right] / \sqrt{1+(a \mid c)^{2}}$} \\
\hline 212 & {$\left[-\frac{1}{2}+\frac{3}{\sqrt{12}}+\frac{2}{\sqrt{3}}(a \mid c)\right] / \sqrt{5+4(a \mid c)^{2}}$} & {$\left[-\frac{1}{2}+\frac{3}{\sqrt{12}}+\frac{2}{\sqrt{3}}(a \mid c)\right] / \sqrt{5+4(a \mid c)^{2}}$} & {$\left[-\sqrt{3}+\frac{2}{\sqrt{3}}(a / c)\right] / \sqrt{5+4(a \mid c)^{2}}$} \\
\hline 122 & {$\left[-\frac{1}{2}+\frac{3}{\sqrt{12}}+\frac{2}{\sqrt{3}}(a \mid c)\right] / \sqrt{5+4(a \mid c)^{2}}$} & {$\left[\frac{1}{2}+\frac{3}{\sqrt{12}}+\frac{2}{\sqrt{3}}(a \mid c)\right] / \sqrt{5+4(a \mid c)^{2}}$} & {$\left[-\sqrt{3}+\frac{2}{\sqrt{3}}(a \mid c)\right] / \sqrt{5+4(a \mid c)^{2}}$} \\
\hline$\overline{2} 12$ & {$\left[-\frac{3}{2}-\frac{1}{\sqrt{12}}+\frac{2}{\sqrt{3}}(a \mid c)\right] / \sqrt{5+4(a \mid c)^{2}}$} & {$\left[\frac{3}{2}-\frac{1}{\sqrt{12}}+\frac{2}{\sqrt{3}}(a \mid c)\right] / \sqrt{5+4(a \mid c)^{2}}$} & {$\left[\frac{1}{\sqrt{3}}+\frac{2}{\sqrt{3}}(a / c)\right] / \sqrt{5+4(a / c)^{2}}$} \\
\hline $2 \overline{1} 2$ & {$\left[\frac{3}{2}+\frac{1}{\sqrt{12}}+\frac{2}{\sqrt{3}}(a \mid c)\right] / \sqrt{5+4(a / c)^{2}}$} & {$\left[-\frac{3}{2}+\frac{1}{\sqrt{12}}+\frac{2}{\sqrt{3}}(a \mid c)\right] / \sqrt{5+4(a \mid c)^{2}}$} & {$\left[-\frac{1}{\sqrt{3} \overline{3}}+\frac{2}{\sqrt{3}}(a / c)\right] / \sqrt{5+4(a / c)^{2}}$} \\
\hline$\overline{1} 22$ & {$\left[-\frac{3}{2}+\frac{1}{\sqrt{12}}+\frac{2}{\sqrt{3}}(a \mid c)\right] / \sqrt{5+4(a \mid c)^{2}}$} & {$\left[\frac{3}{2}+\frac{1}{\sqrt{12}}+\frac{2}{\sqrt{3}}(a \mid c)\right] / \sqrt{5+4(a \mid c)^{2}}$} & {$\left[-\frac{1}{\sqrt{3}}+\bar{V}^{2} \overline{\overline{3}}(a \mid c)\right] / \sqrt{5+4(a \mid c)^{2}}$} \\
\hline $1 \overline{2} 2$ & {$\left[\frac{3}{2}-\frac{1}{\sqrt{12}}+\frac{2}{\sqrt{3}}(a \mid c)\right] / \sqrt{5+4(a \mid c)^{2}}$} & {$\left[-\frac{3}{2}-\frac{1}{\sqrt{12}}+\frac{2}{\sqrt{3}}(a \mid c)\right] / \sqrt{5+4(a / c)^{2}}$} & {$\left[\bar{v}^{1} \overline{\overline{3}}+\frac{2}{\sqrt{3}}(a \mid c)\right] / \sqrt{5+4(a \mid c)^{2}}$} \\
\hline $21 \overline{2}$ & {$\left[\frac{1}{2}-+\frac{3}{\sqrt{12}}-\frac{2}{\sqrt{3}}(a \mid c)\right] / \sqrt{5+4(a \mid c)^{2}}$} & {$\left[-\frac{1}{2}+\frac{3}{\sqrt{12}}-\frac{2}{\sqrt{3}}(a / c)\right] / \sqrt{5+4(a / c)^{2}}$} & {$\left[-\sqrt{3}-\frac{2}{\sqrt{3}}(a \mid c)\right] / \sqrt{5+4(a \mid c)^{2}}$} \\
\hline $12 \overline{2}$ & {$\left[-\frac{1}{2}+\frac{3}{\sqrt{12}}-\frac{2}{\sqrt{3}}(a \mid c)\right] / \sqrt{5+4(a \mid c)^{2}}$} & {$\left[\frac{1}{2}+\frac{3}{\sqrt{\overline{12}}}-\frac{2}{\sqrt{3}}(a \mid c)\right] / \sqrt{5+4(a / c)^{2}}$} & {$\left[-\sqrt{3}-\frac{2}{\sqrt{3}}(a \mid c)\right] / \sqrt{5+4(a \mid c)^{2}}$} \\
\hline 411 & {$\left[\frac{3}{2}+\frac{5}{\sqrt{12}}+\frac{1}{\sqrt{3}}(a \mid c)\right] / \sqrt{17+(a \mid c)^{2}}$} & {$\left[-\frac{3}{2}+\frac{5}{\sqrt{12}}+\frac{1}{\sqrt{3}}(a \mid c)\right] / \sqrt{17+(a \mid c)^{2}}$} & {$\left[-\frac{5}{\sqrt{3}}+\frac{1}{\sqrt{3}}(a / c)\right] / \sqrt{17+(a \mid c)^{2}}$} \\
\hline 141 & {$\left[-\frac{3}{2}+\frac{5}{\sqrt{12}}+\frac{1}{\sqrt{\overline{3}}}(a \mid c)\right] / \sqrt{17+(a \mid c)^{2}}$} & {$\left[\frac{3}{2}+\frac{5}{\sqrt{12}}+\frac{1}{\sqrt{3}}(a \mid c)\right] / \sqrt{17+(a \mid c)^{2}}$} & {$\left[-\frac{5}{\sqrt{\overline{3}}}+\frac{1}{\sqrt{3}}(a \mid c)\right] / \sqrt{17+(a \mid c)^{2}}$} \\
\hline$\overline{4} 11$ & {$\left[-\frac{5}{2}-\frac{3}{\sqrt{12}}+\frac{1}{\sqrt{3}}(a \mid c)\right] / \sqrt{17+(a \mid c)^{2}}$} & {$\left[\frac{5}{2}-\frac{3}{\sqrt{12}}+\frac{1}{\sqrt{3}}(a \mid c)\right] / \sqrt{17+(a \mid c)^{2}}$} & {$\left[\sqrt{3}+\frac{1}{\sqrt{-3}}(a \mid c)\right] / \sqrt{17+(a \mid c)^{2}}$} \\
\hline $4 \overline{1} 1$ & {$\left[\frac{5}{2}+\frac{3}{\sqrt{\mathbf{1 2}}}+\frac{1}{\sqrt{3}}(a \mid c)\right] / \sqrt{17+(a \mid c)^{2}}$} & {$\left[-\frac{5}{2}+\frac{3}{\sqrt{12}}+\frac{1}{\sqrt{3}}(a \mid c)\right] / \sqrt{17+(a \mid c)^{2}}$} & {$\left[-\sqrt{3}+\frac{1}{\sqrt{3}}(a|c\rangle] / \sqrt{17+(a \mid c)^{2}}\right.$} \\
\hline$\overline{1} 41$ & {$\left[-\frac{5}{2}+\frac{3}{\sqrt{12}}+\frac{1}{\sqrt{\overline{3}}}(a \mid c)\right] / \sqrt{17+(a / c)^{2}}$} & {$\left[\frac{5}{2}+\frac{3}{\sqrt{12}}+\frac{1}{\sqrt{3}}(a \mid c)\right] / \sqrt{17+(a \mid c)^{2}}$} & {$\left[-\sqrt{3}+\frac{1}{\sqrt{3}}(a \mid c)\right] / \sqrt{17+(a \mid c)^{2}}$} \\
\hline $1 \overrightarrow{4} 1$ & {$\left[\frac{5}{2}--\frac{3}{\sqrt{12}}+\frac{1}{\sqrt{3}}(a \mid c)\right] / \sqrt{17+(a \mid c)^{2}}$} & {$\left[-\frac{5}{2}-\frac{3}{\sqrt{12}}+\frac{1}{\sqrt{3}}(a \mid c)\right] / \sqrt{17+(a \mid c)^{2}}$} & {$\left[\sqrt{3}+\frac{1}{\sqrt{3}}(a \mid c)\right] / \sqrt{17+(a \mid c)^{2}}$} \\
\hline $41 \overline{1}$ & {$\left[\frac{3}{2}+\frac{5}{\sqrt{12}}-\frac{1}{\sqrt{3}}(a \mid c)\right] / \sqrt{17+(a \mid c)^{2}}$} & {$\left[-\frac{3}{2}+\frac{5}{\sqrt{12}}-\frac{1}{\sqrt{3}}(a \mid c)\right] / \sqrt{17+(a \mid c)^{2}}$} & {$\left[-\frac{5}{\sqrt{3}}-\frac{1}{\sqrt{3}}(a / c)\right] / \sqrt{17+(a / c)^{2}}$} \\
\hline 14I & {$\left[-\frac{3}{2}+\frac{5}{\sqrt{12}}-\frac{1}{\sqrt{3}}(a \mid c)\right] / \sqrt{17+(a / c)^{2}}$} & {$\left[\frac{3}{2}+\frac{5}{\sqrt{12}}-\frac{1}{\sqrt{3}}(a \mid c)\right] / \sqrt{17+(a \mid c)^{2}}$} & {$\left[-\frac{5}{\sqrt{3}}-\frac{1}{\sqrt{3}}(a / c)\right] / \sqrt{17+(a / c)^{2}}$} \\
\hline 331 & {$\left[\frac{6}{\sqrt{12}}+\frac{1}{\sqrt{3}}(a \mid c)\right] / \sqrt{18+(a \mid c)^{2}}$} & {$\left[\frac{6}{\sqrt{12}}+\frac{1}{\sqrt{3}}(a \mid c)\right] / \sqrt{18+(a \mid c)^{2}}$} & {$\left[-2 \sqrt{3}+\frac{1}{\sqrt{3}}(a \mid c)\right] / \sqrt{18+(a / c)^{2}}$} \\
\hline$\overline{3} 31$ & {$\left[-3+\frac{1}{\sqrt{3}}(a / c)\right] / \sqrt{18+(a \mid c)^{2}}$} & {$\left[3+\frac{1}{\sqrt{3}}(a \mid c)\right] / \sqrt{18+(a \mid c)^{2}}$} & $\frac{1}{\sqrt{3}}(a \mid c) / \sqrt{18+(a \mid c)^{2}}$ \\
\hline $3 \overline{3} 1$ & {$\left[3+\frac{1}{\sqrt{3}}(a / c)\right] / \sqrt{18+(a / c)^{2}}$} & {$\left[-3+\frac{1}{\sqrt{3}}(a \mid c)\right] / \sqrt{18+(a \mid c)^{2}}$} & $\frac{1}{\sqrt{\overline{3}}}(a \mid c) / \sqrt{18+(a \mid c)^{2}}$ \\
\hline $33 \overline{1}$ & {$\left[\frac{6}{\sqrt{12}}-\frac{1}{\sqrt{3}}(a \mid c)\right] / \sqrt{18+(a / c)^{2}}$} & {$\left[\frac{6}{\sqrt{12}}-\frac{1}{\sqrt{3}}(a / c)\right] / \sqrt{18+(a / c)^{2}}$} & {$\left[-2 \sqrt{3}-\frac{1}{\sqrt{3}}(a \mid c)\right] / \sqrt{18+(a \mid c)^{2}}$} \\
\hline
\end{tabular}


Table 5 Direction cosines of $\sigma\{h k l\}$ referred to gamma principal axes, as calculated by using orientation relationship $B$.

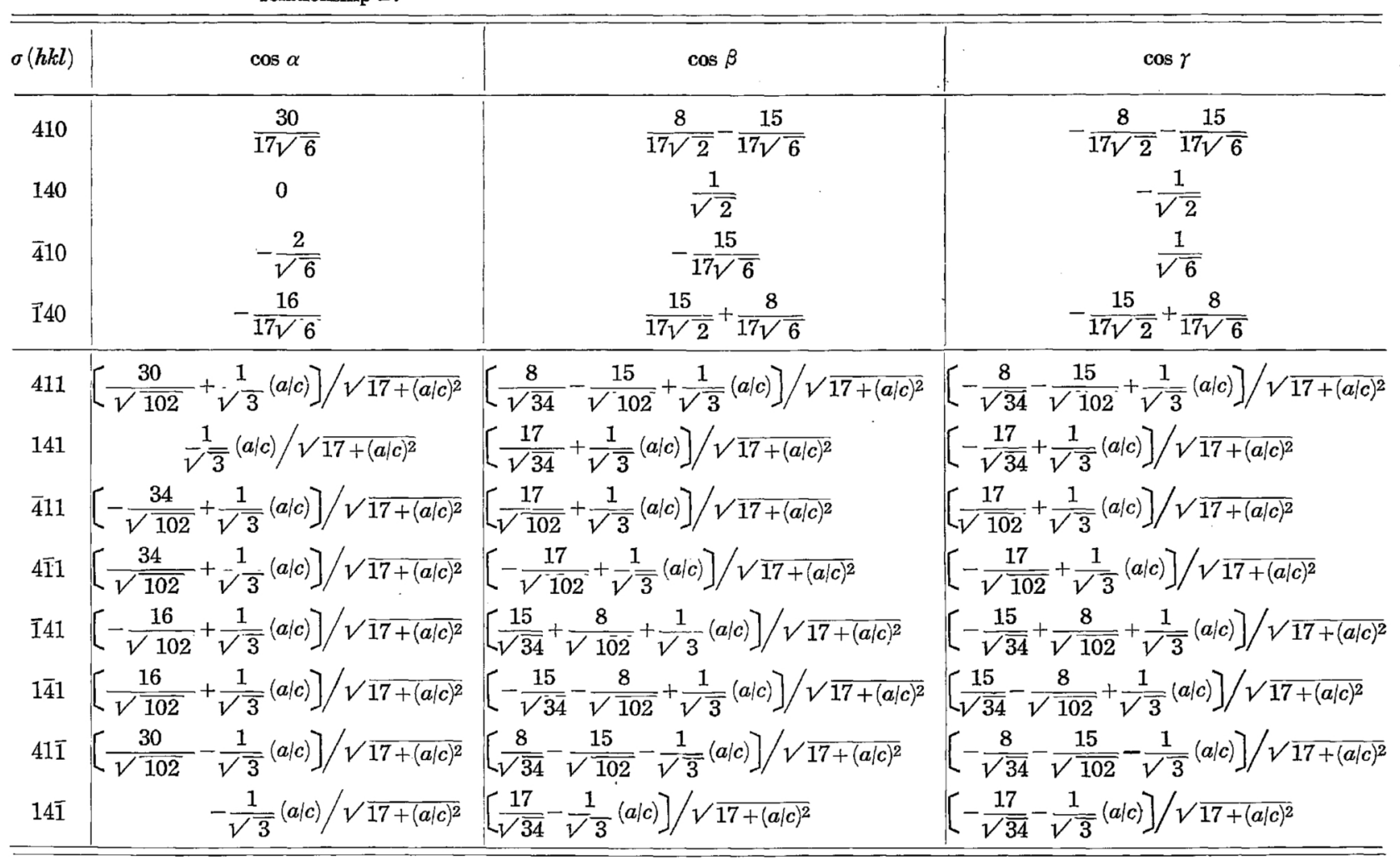

The number of variants is 12 in this case. The direction cosines of the planes $\{140\}$ of sigma crystal is calculated as shown in Table 6.

Table 6 Direction cosines of $\sigma\{k h l\}$ referred to gamma principal axes, as calculated by using orientation relationship $\mathrm{C}$.

\begin{tabular}{c|c|c|c}
\hline \hline$\sigma(h k l)$ & $\cos \alpha$ & $\cos \beta$ & $\cos \gamma$ \\
\hline 410 & $\frac{1}{\sqrt{17}}\left(-\frac{5}{\sqrt{6}}-\frac{3}{2}\right)$ & $\frac{1}{\sqrt{17}}\left(-\frac{5}{\sqrt{6}}+\frac{3}{2}\right)$ & $-\frac{5}{\sqrt{102}}$ \\
140 & $\frac{1}{\sqrt{17}}\left(-\frac{5}{\sqrt{6}}+\frac{3}{2}\right)$ & $\frac{1}{\sqrt{17}}\left(-\frac{5}{\sqrt{6}}-\frac{3}{2}\right)$ & $-\frac{5}{\sqrt{102}}$ \\
$\overline{4} 10$ & $\frac{1}{\sqrt{17}}\left(\frac{3}{\sqrt{6}}+\frac{5}{2}\right)$ & $\frac{1}{\sqrt{17}}\left(\frac{3}{\sqrt{6}}-\frac{5}{2}\right)$ & $\frac{3}{\sqrt{102}}$ \\
$\overline{1} 40$ & $\frac{1}{\sqrt{17}}\left(-\frac{3}{\sqrt{6}}+\frac{5}{2}\right)$ & $\frac{1}{\sqrt{17}}\left(-\frac{3}{\sqrt{6}}-\frac{5}{2}\right)$ & $-\frac{3}{\sqrt{102}}$ \\
\hline \hline
\end{tabular}

3. $\sigma(\overline{\mathbf{1} 40})$ and $\gamma(\mathbf{1 1 1})$

As indicated by $A^{\prime}, B^{\prime}, A^{\prime}$, in Fig. 1 , the spacing of $\sigma(\overline{1} 40)$ is nearly the same as that of $\sigma(330)$ and the atomic arrangement in $\sigma(\overline{140)}$ is essentially not greatly different from that of $\sigma(110)$. The possible orientation relationship would be :

$$
\begin{aligned}
& \sigma(\overline{140)} \| \gamma(111) \\
& \sigma[001] \| \gamma[\overline{\mathrm{I}} 2] .
\end{aligned}
$$

As seen from Fig. 1, $\sigma[410]$ and $\sigma[\overline{1} 10]$ correspond to two edges of a hexagonal net in $\sigma(001)$, respectively. Hence, there seems to be not great difference between orientation relationships $\mathrm{C}$ and $\mathrm{E}$. Therefore, no further consideration about relationship $\mathrm{E}$ is made here.

\section{Assumed Orientation Relationships and Observed Positions of Interference Spots}

The expected positions of the interference spots in the oscillation photograph can be calculated on the assumption of an orientation relationship, similarly as given by one of the authors ${ }^{(9)}$.

\section{Rotation about $\gamma[001]$}

When the crystal is rotated by an angle $\Theta$ from one of the principal directions (Fig. 5) and the reflection from a net plane $(\cos \alpha, \cos \beta, \cos \gamma)$ of a sigma crystal occurs at a Bragg angle $\theta$, there is the following relation among $\Theta, \theta, \cos \alpha, \cos \beta$ and $\cos \gamma$ :

$$
\sin \Theta=\frac{ \pm \sin \theta \cos \beta \pm \cos \alpha \sqrt{\cos ^{2} \alpha+\cos ^{2} \beta-\sin ^{2} \theta}}{\cos ^{2} \alpha+\cos ^{2} \beta}
$$

The position $\delta$ of the spot is given by

$$
\cos \delta=\frac{\cos \gamma}{\cos \theta}
$$

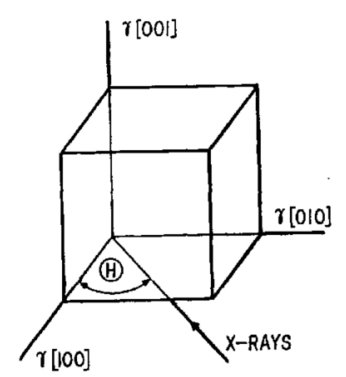

Fig. 5 Diagram showing the relation between incident beams and principal axes of gamma crystal in the case of rotation about $\gamma[001]$ axis.

\section{Rotation about $\gamma[\mathbf{0 1 1}]$}

In this case (Fig. 6) the corresponding relations are as follows : 


$$
\sin \Theta=\frac{ \pm \sin \theta \frac{1}{\sqrt{2}}(\cos \beta+\cos \gamma)}{\cos ^{2} \alpha+\frac{1}{\cos ^{2} \alpha+\frac{1}{2}(\cos \beta+\cos \gamma)^{2}-\sin ^{2} \theta}}
$$

and

$$
\cos \delta=\frac{1}{\cos \theta}\left(-\frac{1}{\sqrt{2}} \cos \beta+\frac{1}{\sqrt{2}} \cos \gamma\right)
$$

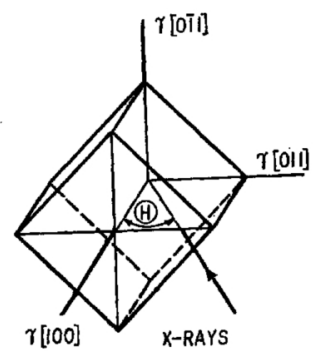

Fig. 6 Diagram showing the relation between incident beams and principal axes of gamma crystal in the case of rotation about $\gamma[0 \overline{1} 1]$ axis.

Using equation (9) along with the values of the direction cosines given in Tables 4,5 and 6 and of their equivalents, we calculate the values of position $\delta$ of $\sigma\{140\}$ interference spots in the case of complete rotation about $\gamma[0 \overline{1} 1]$ axis, on the assumption of each of orientation relationships $\mathrm{A}, \mathrm{B}$ and $\mathrm{C}$. These calculated values are compared with the experimental values (in Table 1), in order to find out which orientation relationship best fits the experimental results.

In this calculation the wave length of $\mathrm{Cr}-K \alpha, 2.8909$ $\AA$, and the lattice constants of the sigma phase, $a_{0}=$ $8.779 \AA, c_{0}=4.533 \AA$, were used.

As seen from Table 1 and Fig. 7, the values of $\delta$ calculated on the assumption of orientation relation-

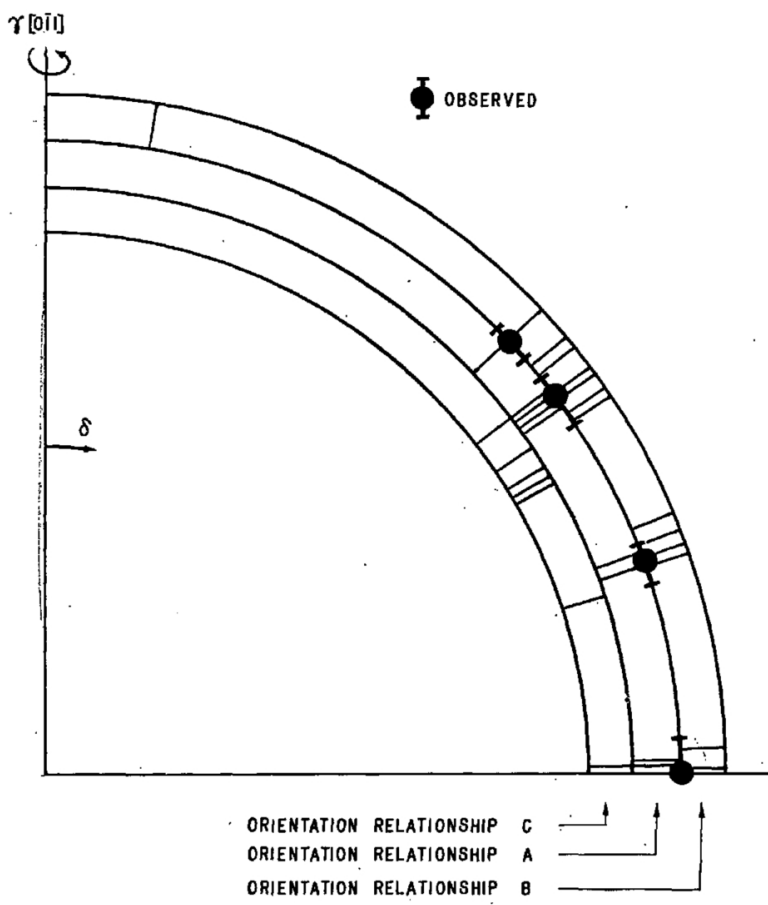

Fig. 7 Comparison between calculated and observed values of $\delta$ of $\sigma\{140\}$ spots. (see Photo. 3, $r$ [0피] rotation axis, $\mathrm{Cr}-K \alpha$ radiation) ship A are in good agreement with the observed $\delta$ values, while the calculated $\delta$ values in orientation relationship $\mathrm{C}$ are not. In the case of orientation relationship $B$, agreement between the calculation and experiment is not bad, but it does not seem as good as in the case of orientation relationship A. As already shown in Fig. 4 (a) and (b), the difference between two orientation relationships $A$ and $B$ is only $58^{\prime}$. The next thing to do is to choose the one out of these two relationships. For this purpose, the $\delta$ values of $\sigma\{411\}$ spots, which have very high intensity, calculated from relationships A and B were compared to the experimental values (in Table 1) obtained from Photo. 3 in the case of $\gamma[0 \overline{1} 1]$-rotation with $\mathrm{Cr}-K \alpha$ radiation. As seen from Table 1 , the $\delta$ values calculated from two relationships $A$ and $B$ are both in good agreement with the observed values, within the limit of the experimental error. So far the calculated $\delta$ values were compared to the observed ones as obtained from $\mathrm{Cr}-K \alpha$ rotation photographs in which interference spots with $\delta$ values less than about $30^{\circ}$ are not visible in the photographs, due to the geometry of the camera used. To overcome this difficulty, the rotation photograph about $\gamma[0 \overline{1} 1]$ axis with $\mathrm{Mo}-K \alpha$

\begin{tabular}{|c|c|c|}
\hline \multirow[t]{2}{*}{$\delta_{\text {obs. }}$} & \multicolumn{2}{|c|}{$\delta_{\text {calc. }}$} \\
\hline & Orientation-relationship A & Orientation relationship B \\
\hline $23^{\circ}$ & $22^{\circ} 58^{\prime}$ & $\begin{array}{l}22^{\circ} 56^{\prime} \\
23^{\circ} 1^{\prime}\end{array}$ \\
\hline $28^{\circ}$ & $27^{\circ} 48^{\prime}$ & $27^{\circ} 47^{\prime}$ \\
\hline $36^{\circ}$ & $\left.\begin{array}{rr}35^{\circ} & 8^{\prime} \\
36^{\circ} & 1^{\prime} \\
36^{\circ} & 22^{\prime} \\
37^{\circ} & 51^{\prime}\end{array}\right\}$ & $\left.\begin{array}{lr}34^{\circ} & 42^{\prime} \\
35^{\circ} & 34^{\prime} \\
35^{\circ} & 39^{\prime} \\
36^{\circ} & 28^{\prime} \\
37^{\circ} & 6^{\prime} \\
37^{\circ} & 56^{\prime} \\
38^{\circ} & 36\end{array}\right\}$ \\
\hline $52^{\circ}$ & $\left.\begin{array}{l}51^{\circ} 14^{\prime} \\
52^{\circ} 20^{\prime}\end{array}\right\}$ & $\left.\begin{array}{ll}51^{\circ} & 10^{\prime} \\
51^{\circ} & 46^{\prime} \\
52^{\circ} & 54^{\prime}\end{array}\right\}$ \\
\hline $62^{\circ}$ & $\begin{array}{l}61^{\circ} 43^{\prime} \\
63^{\circ} 27^{\prime}\end{array}$ & $\left.\begin{array}{l}60^{\circ} 51^{\prime} \\
62^{\circ} 35^{\prime} \\
64^{\circ} 19^{\prime}\end{array}\right\}$ \\
\hline $69^{\circ}$ & $\left.\begin{array}{l}68^{\circ} 47^{\prime} \\
69^{\circ} 53^{\prime}\end{array}\right\}$ & $\left.\begin{array}{ll}68^{\circ} & 14^{\prime} \\
69^{\circ} & 20^{\prime} \\
70^{\circ} & 25^{\prime}\end{array}\right\}$ \\
\hline $79^{\circ}$ & $79^{\circ} 43^{\prime}$ & $\left.\begin{array}{l}79^{\circ} 18^{\prime} \\
79^{\circ} 42^{\prime}\end{array}\right\}$ \\
\hline $84^{\circ}$ & $\left.\begin{array}{rr}83^{\circ} & 35^{\prime} \\
84^{\circ} & 5^{\prime} \\
84^{\circ} 33^{\prime}\end{array}\right\}$ & $\left.\begin{array}{rr}83^{\circ} & 20^{\prime} \\
83^{\circ} & 50^{\prime} \\
84^{\circ} & 6^{\prime} \\
84^{\circ} & 22^{\prime} \\
85^{\circ} & 0^{\prime}\end{array}\right\}$ \\
\hline $90^{\circ}$ & $89^{\circ} 7^{\prime}$ & $\left.\begin{array}{rr}88^{\circ} & 14^{\prime} \\
88^{\circ} & 38^{\prime} \\
90^{\circ} & 0^{\prime}\end{array}\right\}$ \\
\hline
\end{tabular}

Table 7 Comparison between experimental and calculated $\delta$ values of $\sigma\{411\}$ spots in a rotation photograph about the $\gamma[0 \overline{1} 0]$ axis, Mo- $K \alpha$ radiation, flat film (Photo. 4).

radiation (Photo. 4), was used to make a comparison between the observed and calculated $\delta$ values. In this case the wave length of Mo- $K \alpha, 0.71069 \AA$, was used. Table 7 shows $\delta$ values calculated from orientation 
relationships $\mathrm{A}$ and $\mathrm{B}$, and the observed $\delta$ values obtained from Photo. $4^{*}$. From this table it is noted that the $\delta$ values calculated from both of the relationships are in good agreement with the observed ones.

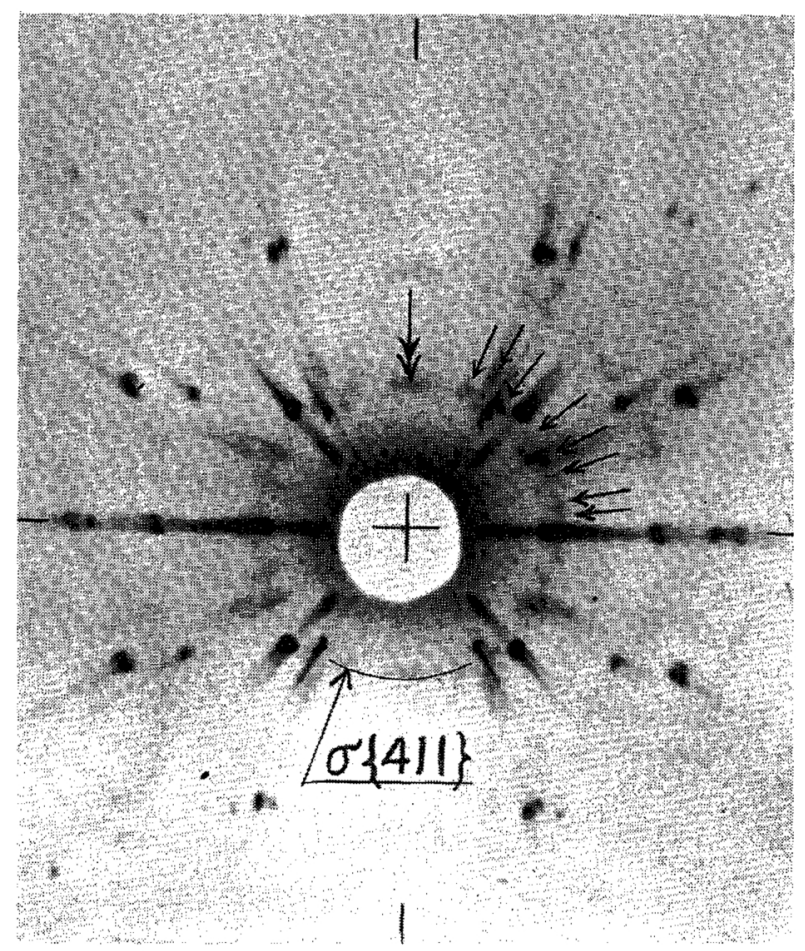

Photo. 4 Rotation photogiaph about $r[0 \overline{1} 1]$ axis, Mo- $K \alpha$ radiation. Specimen: $815^{\circ} \mathrm{C}, 218 \mathrm{hrs}$; crystal-film distance $41 \mathrm{~mm}$. Single arrow $(\downarrow)$ indicating $\sigma\{411\}$ spots, double ariow $(\Downarrow) \sigma\{212\}, \sigma\{202\}$ spots.

Therefore, we can say that both orientation relationships A and B can explain the experimental result within the limit of experimental error. Since the difference between two relationships is so small that it was not possible to tell, under the accuracy of the present experiment, which relationship fits better the experimental result, hereafter we shall only consider relationship A. In Table 1 are listed the calculated $\delta$ values of $\sigma\{330\}, \sigma\{202\}, \sigma\{212\}$, and $\sigma\{331\}$ interference spots and their corresponding experimental values obtained from the rotation photograph about $\gamma[0 \overline{1} 1]$ axis with $\mathrm{Cr}-K \alpha$ (Photo. 3 ). In Table 2 are listed the calculated $\delta$ values of $\sigma\{140\}, \sigma\{330\}$, $\sigma\{202\}, \sigma\{212\}, \sigma\{411\}$, and $\sigma\{331\}$ interference spots and their corresponding experimental values obtained from the rotation photograph about $\gamma[001]$ axis with $\mathrm{Cr}-K_{\alpha}$ (Photo. 2). As seen from Tables 1 and 2 , the calculated $\delta$ values are in good agreement with the corresponding experimental values within the limit of the experimental error.

Finally, let us check whether a spot appears or not at the position $\delta$ for a defined orientation $\Theta$ of the specimen, these two quantities $\Theta$ and $\delta$ being predicted by orientation relationship $A$. The values of $\Theta$ and $\delta$, for $\operatorname{Cr}-K \alpha$ radiation, as calculated from orientation relationship A using equations (6), (7), (8) and (9) are given in Tables 8 and 9. For one position of the $\Theta$ value of the specimen, two spots appear in

* The spots indicated by a double ariow ( $\Downarrow$ ) coriespond to $\sigma\{212\}\left(\delta \sim 0^{\circ}, \delta=4^{\circ} 9^{\prime}\right)$ and $\sigma\{202\}\left(\delta=4^{\circ} 31^{\prime}\right)$ spots. pair at $\delta$ and $180^{\circ}-\delta$; in the above tables they are distinguished as upper and lower spots at $\delta$. Photo. 5 (a), (b), $\cdots \cdots$, and (d) were taken by oscillating the specimen by $2^{\circ}$ about $\gamma[001]$ axis near the angle $\Theta$

Table $8 \Theta$ and $\delta$ values for $\sigma\{140\}$ and $\sigma\{330\}$ spots as calculated by using equations $(6)$ and $(7)$ in the case of rotation about $\gamma[001]$ axis, $\mathrm{Cr}-K \alpha$ radiation.

\begin{tabular}{|c|c|c|c|}
\hline \multirow{2}{*}{$\begin{array}{c}\text { Angle of rotation } \\
\text { from } \gamma[100] \\
\theta\end{array}$} & \multirow{2}{*}{$\begin{array}{c}\text { Position } \\
\text { of spot } \\
\delta\end{array}$} & \multicolumn{2}{|c|}{ Reflecting plane of $\sigma$ crystal } \\
\hline & & Upper spot & Lower spot \\
\hline $\begin{array}{r}8^{\circ} 38^{\prime} \\
10^{\circ} 13^{\prime} \\
11^{\circ} 54^{\prime} \\
13^{\circ} 0^{\prime} \\
22^{\circ} 0^{\prime} \\
25^{\circ} 21^{\prime} \\
26^{\circ} 54^{\prime} \\
27^{\circ} 45^{\prime} \\
38^{\circ} 41^{\prime} \\
40^{\circ} 1^{\prime} \\
40^{\circ} 56^{\prime} \\
42^{\circ} 13^{\prime}\end{array}$ & $\begin{array}{rr}+61^{\circ} & 57^{\prime} \\
+60^{\circ} & 7^{\prime} \\
-89^{\circ} & 4^{\prime} \\
-89^{\circ} & 4^{\prime} \\
+14^{\circ} & 26^{\prime} \\
+14^{\circ} & 26^{\prime} \\
-61^{\circ} & 57^{\prime} \\
-60^{\circ} & 7^{\prime} \\
-32^{\circ} & 8^{\prime} \\
-33^{\circ} & 51^{\prime} \\
-32^{\circ} & 8^{\prime} \\
-33^{\circ} & 51^{\prime}\end{array}$ & $\begin{array}{l}\mathbf{A}(\mathbf{9})(\overline{1} 40) \\
\mathbf{A}(\mathbf{8})(410) \\
\mathbf{A}(3)(410) \\
\mathbf{A}(11) \\
\mathbf{A}(12) \\
\mathbf{A}(11)(\overline{410}) \\
\mathbf{A}(11) \\
\mathbf{A}(10)(\overline{1} 10) \\
\mathbf{A}(4)(410) \\
\mathbf{A}(10)(140) \\
\mathbf{A}(12)(410) \\
\mathbf{A}(6)(140)\end{array}$ & $\begin{array}{l}\mathbf{A}(4)(\overline{1} 40) \\
\mathbf{A}(6)(\overline{4} 10) \\
\mathbf{A}(12)(140) \\
\mathbf{A}(2)(140) \\
\mathbf{A}(3)(\overline{1} 40) \\
\mathbf{A}(2)(\overline{4} 10) \\
\mathbf{A}(2)(\overline{1} 40) \\
\mathbf{A}(1)(\overline{4} 10) \\
\mathbf{A}(9)(410) \\
\mathbf{A}(1)(410) \\
\mathbf{A}(3)(140) \\
\mathbf{A}(8)(140)\end{array}$ \\
\hline \multicolumn{4}{|l|}{$\sigma\{330\}$} \\
\hline \multirow{2}{*}{$\begin{array}{c}\text { Angle of rotation } \\
\text { from } \gamma[100] \\
\Theta\end{array}$} & \multirow{2}{*}{$\begin{array}{c}\text { Positicn } \\
\text { of spot } \\
\delta\end{array}$} & \multicolumn{2}{|c|}{ Reflecting plane of $\sigma$ crystal } \\
\hline & & Upper spot & Lowar spot \\
\hline $\begin{array}{l}10^{\circ} 46^{\prime} \\
11^{\circ} 23^{\prime} \\
26^{\circ} 6^{\prime} \\
28^{\circ} 29^{\prime} \\
38^{\circ} 29^{\prime}\end{array}$ & $\begin{array}{rr}+60^{\circ} & 39^{\prime} \\
-90^{\circ} & 0^{\prime} \\
-60^{\circ} & 39^{\prime} \\
+11^{\circ} & 21^{\prime} \\
-31^{\circ} & 53^{\prime}\end{array}$ & $\begin{array}{c}\mathbf{A}(7)(330) \\
\mathbf{A}(1)(\overline{3} 30) \\
\mathbf{A}(\mathbf{1 2})(330) \\
\mathbf{A}(10)(330) \\
\mathbf{A}(5)(\overline{3} 30) \\
\mathbf{A}(11)(\overline{3} 30)\end{array}$ & $\begin{array}{l}\mathbf{A}(5)(330) \\
\mathbf{A}(10)(\overline{3} 30) \\
\mathbf{A}(\mathbf{3})(330) \\
\mathbf{A}(1)(330) \\
\mathbf{A}(2)(\overline{3} 30) \\
\mathbf{A}(7)(\overline{3} 30)\end{array}$ \\
\hline
\end{tabular}

Table $9 \Theta$ and $\delta$ values for $\sigma\{140\}$ and $\sigma\{330\}$ spots as calculated by using equations ( 8 ) and (9) in the case of rotation about $\gamma[0 \overline{1} 1]$ axis, $\mathrm{Cr}-K \alpha$ radiation.

$\sigma\{140\}$

\begin{tabular}{|c|c|c|c|}
\hline \multirow{2}{*}{$\begin{array}{c}\text { Angle of rotation } \\
\text { from } \gamma[100] \\
\Theta\end{array}$} & \multirow{2}{*}{$\begin{array}{c}\text { Position } \\
\text { of spot } \\
\delta\end{array}$} & \multicolumn{2}{|c|}{ Reflecting plane of $\sigma$ crystal } \\
\hline & & Upper spot & Lower spot \\
\hline $\begin{array}{rr}8^{\circ} 6^{\prime} \\
9^{\circ} 37^{\prime} \\
15^{\circ} 33^{\prime} \\
15^{\circ} 59^{\prime} \\
16^{\circ} 52^{\prime} \\
17^{\circ} 20^{\prime} \\
22^{\circ} 16^{\prime} \\
31^{\circ} 40^{\prime} \\
33^{\circ} 15^{\prime} \\
47^{\circ} 44^{\prime} \\
50^{\circ} 5^{\prime} \\
58^{\circ} 23^{\prime} \\
60^{\circ} 18^{\prime} \\
85^{\circ} 46^{\prime} \\
86^{\circ} 32^{\prime} \\
87^{\circ} 12^{\prime} \\
87^{\circ} 24^{\prime} \\
88^{\circ} 10^{\prime}\end{array}$ & $\begin{array}{l}+69^{\circ} 23^{\prime} \\
+70^{\circ} 36^{\prime} \\
-54^{\circ} 4^{\prime} \\
-52^{\circ} 26^{\prime} \\
-54^{\circ} 53^{\prime} \\
-53^{\circ} 15^{\prime} \\
-88^{\circ} 51^{\prime} \\
+89^{\circ} 20^{\prime} \\
+89^{\circ} 20^{\prime} \\
-46^{\circ} 50^{\prime} \\
-46^{\circ} 50^{\prime} \\
+70^{\circ} 36^{\prime} \\
+69^{\circ} 23^{\prime} \\
-53^{\circ} 15^{\prime} \\
-52^{\circ} 26^{\prime} \\
+88^{\circ} 51^{\prime} \\
-54^{\circ} 53^{\prime} \\
-54^{\circ} 4^{\prime}\end{array}$ & $\begin{array}{l}\mathbf{A}(12)(\overline{4} 10) \\
\mathbf{A}(11)(\overline{1} 40) \\
\mathbf{A}(12)(140) \\
\mathbf{A}(\mathbf{3})(410) \\
\mathbf{A}(2)(140) \\
\mathbf{A}(11)(410) \\
\mathbf{A}(2)(\overline{1} 40) \\
\mathbf{A}(11)(140) \\
\mathbf{A}(7)(140) \\
\mathbf{A}(11)(\overline{4} 10) \\
\mathbf{A}(10)(\overline{1} 40) \\
\mathbf{A}(7)(\overline{1} 40) \\
\mathbf{A}(9)(\overline{4} 10) \\
\mathbf{A}(10)(140) \\
\mathbf{A}(4)(410) \\
\mathbf{A}(1)(\overline{4} 10) \\
\mathbf{A}(6)(140) \\
\mathbf{A}(12)(410)\end{array}$ & 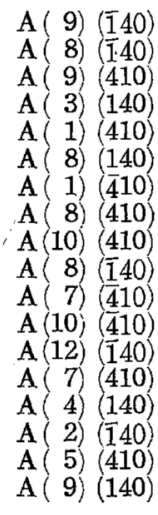 \\
\hline
\end{tabular}

\begin{tabular}{|c|c|c|c|}
\hline \multicolumn{4}{|l|}{$\sigma\{330\}$} \\
\hline \multirow{2}{*}{$\begin{array}{c}\text { Angle of rotation } \\
\text { from } \gamma[100] \\
\Theta\end{array}$} & \multirow{2}{*}{$\begin{array}{c}\text { Position } \\
\text { of spot } \\
\delta\end{array}$} & \multicolumn{2}{|c|}{ Reflecting plane of $\sigma$ crystal } \\
\hline & & Upper spot & Lower spot \\
\hline $10^{\circ} 5^{\prime}$ & $+69^{\circ} 43^{\prime}$ & \multirow{5}{*}{$\begin{array}{c}\mathbf{A}(10)(330) \\
\mathbf{A}(\mathbf{1})(\overline{3} 30) \\
\mathbf{A}(10)(\overline{3} 30) \\
\mathbf{A}(3) \\
\mathbf{A}(9)(\overline{3} 30) \\
\mathbf{A}(12)(330) \\
\mathbf{A}(\mathbf{8})(330) \\
\mathbf{A}(5)(\overline{3} 30) \\
\mathbf{A}(11)(\overline{3} 30) \\
\mathbf{A}(3)\end{array}$} & \multirow{2}{*}{ 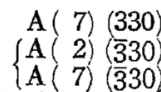 } \\
\hline $15^{\circ} 0^{\prime}$ & $-53^{\circ} 6^{\prime}$ & & \\
\hline $\begin{array}{l}21^{\circ} 8^{\prime} \\
33^{\circ} 37^{\prime} \\
47^{\circ} 19^{\prime} \\
60^{\circ} 34^{\prime}\end{array}$ & $\begin{array}{ll}-90^{\circ} & 0^{\prime} \\
+90^{\circ} & 0^{\prime} \\
-46^{\circ} & 7^{\prime} \\
+69^{\circ} & 43^{\prime}\end{array}$ & & $\begin{array}{l}330) \\
(12)(\overline{3} 30) \\
A(9)(330) \\
A(11)(330)\end{array}$ \\
\hline $85^{\circ} 32^{\prime}$ & $-53^{\circ} \quad 6^{\prime}$ & & $\left\{\begin{array}{l}\mathrm{A}(6) \\
\mathrm{A}\end{array}\right.$ \\
\hline $88^{\circ} 22^{\prime}$ & $+90^{\circ} 0^{\prime}$ & & \\
\hline
\end{tabular}




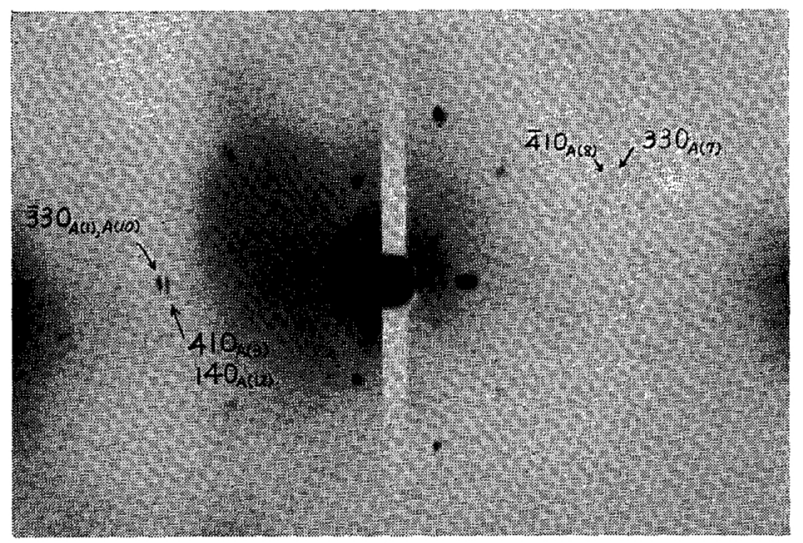

(a)

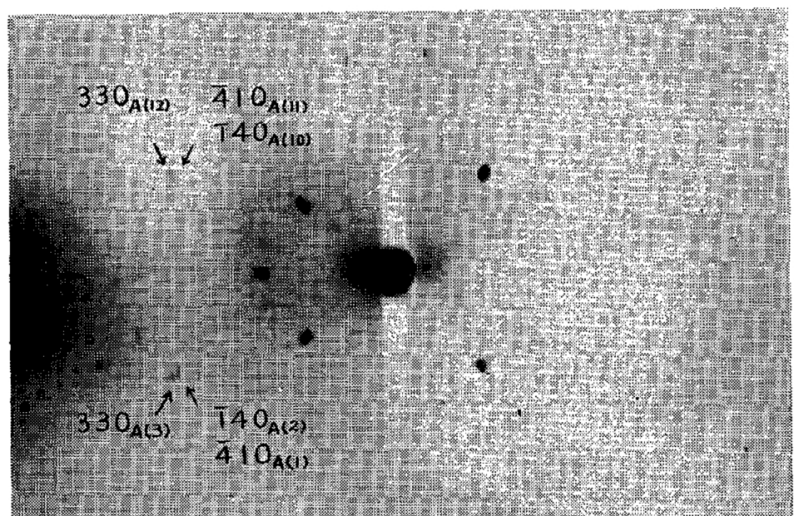

(b)

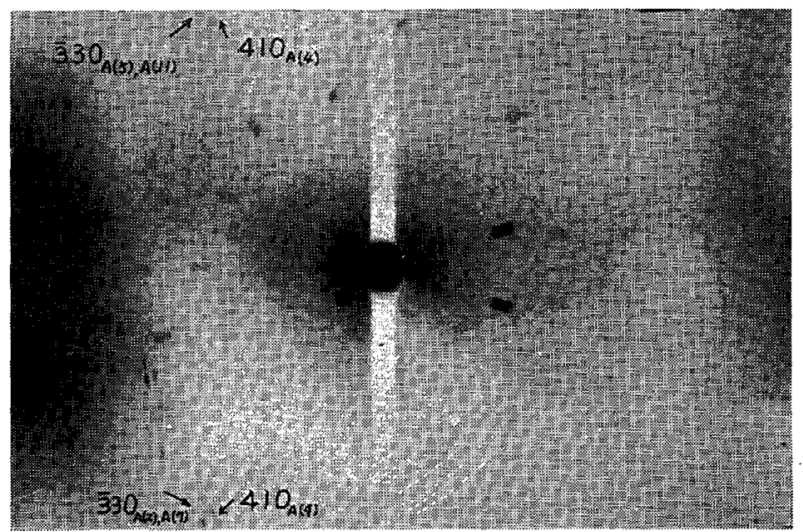

(c)

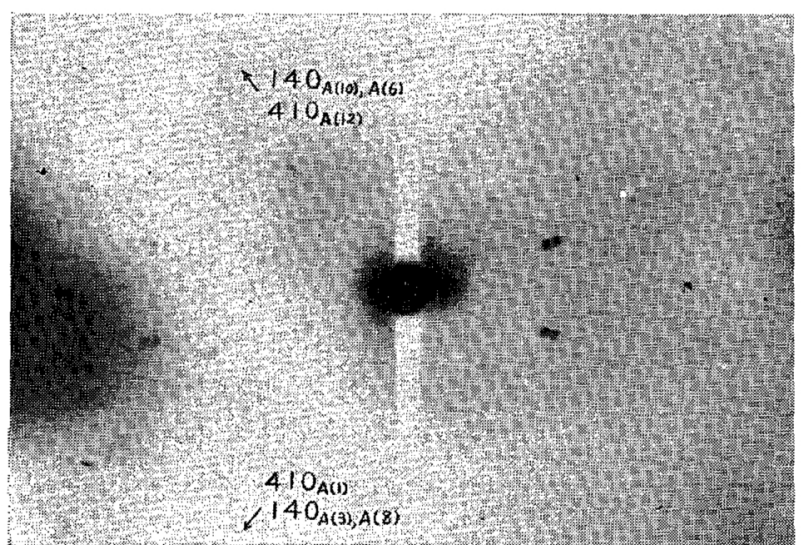

(d)

Photo. 5 Oscillation photographs about $\gamma[001]$ axis, $\mathrm{Cr}-\mathrm{K} \alpha$ radiation. Specimen : $815^{\circ} \mathrm{C}, 218 \mathrm{hrs}$; camera diameter $70 \mathrm{~mm}$.
(a) $\Theta=10.0^{\circ} \sim 11.7^{\circ}$
(b) $\Theta=26.3^{\circ} \sim 28.3^{\circ}$
(c) $\Theta=36.6^{\circ} \sim 38.6^{\circ}$
(d) $\Theta=40.3^{\circ} \sim 42.0^{\circ}$

given in the first column of Table 8. Photo. 6 (a), (b), $\cdots$, and $(h)$ are oscillation photographs about $\gamma[0 \overline{1} 1]$ axis taken in the similar way. All spots appearing in each of these patterns are found to correspond to those expected from the calculation; thus the validity of orientation relationship A has been confirmed. Accordingly, orientation relationship B may be valid as already described. Therefore, the orientation relationships between gamma and sigma phases in this alloy are :

$$
\begin{aligned}
& \gamma(111) \| \sigma(001) \\
& \gamma[\overline{1} 10] \| \sigma[\overline{1} 10], \\
& \gamma(111) \| \sigma(001) \\
& \gamma[01 \overline{1}] \| \sigma[140] .
\end{aligned}
$$

or

\section{Discussion}

On the basis of the orientation relationships established by the present experiment, we now consider the mechanism of the sigma formation from the f.c.c. gamma matrix. Since the difference between two orientation relationships $A$ and $B$ is less than $1^{\circ}$, they will not make much difference in considering the mechanism of sigma formation. A projection on (111) plane of the atomic arrangement of the gamma matrix is shown in Fig. 8, in which successive (111) planes
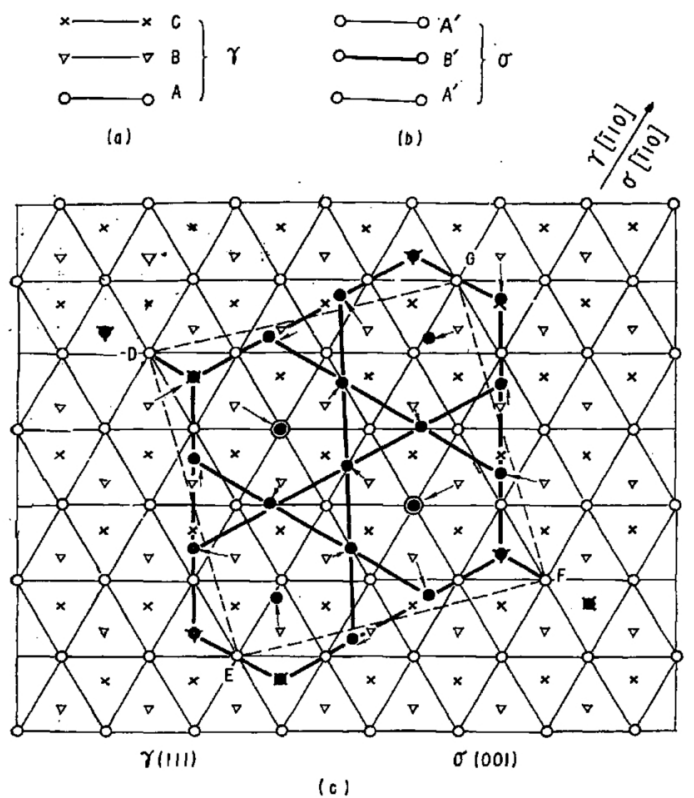

Fig. 8 Illustration of the process of sigma formation in gamma matrix.

are denoted by $A, B, C, A, \cdots$. To construct the atomic arrangement of the sigma structure in accordance with the orientation relationships, let us start with $A$ layer of the gamma matrix with the stacking of atoms described above. In this case the atomic arrangement in the basal plane $\left(A^{\prime}\right)$ of a sigma unit cell can easily be obtained without any movement of atoms, except the atoms at the center of each hexagon which must be shifted in c-direction by $-c_{0} / 4$. The atomic arrangement of the second layer $\left(B^{\prime}\right)$ of the sigma unit cell may be constructed by moving the atoms in $B$ layer $(\gamma(111))$ as indicated by 


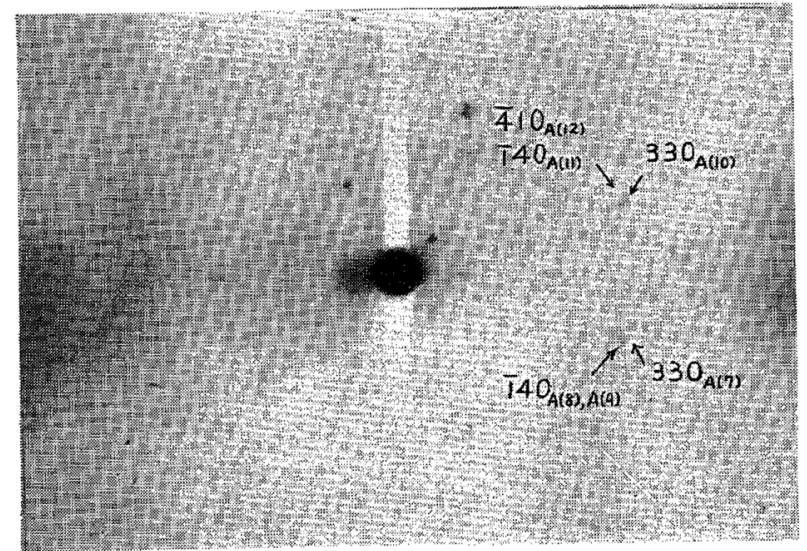

(a)

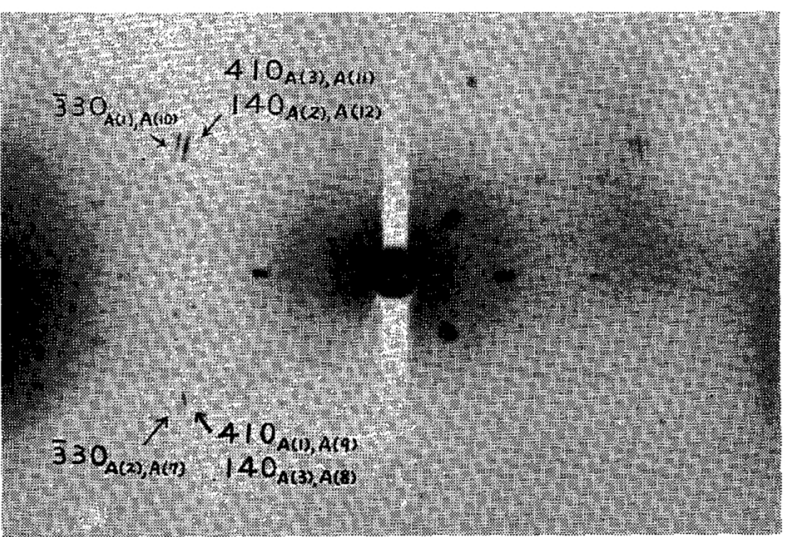

(b)

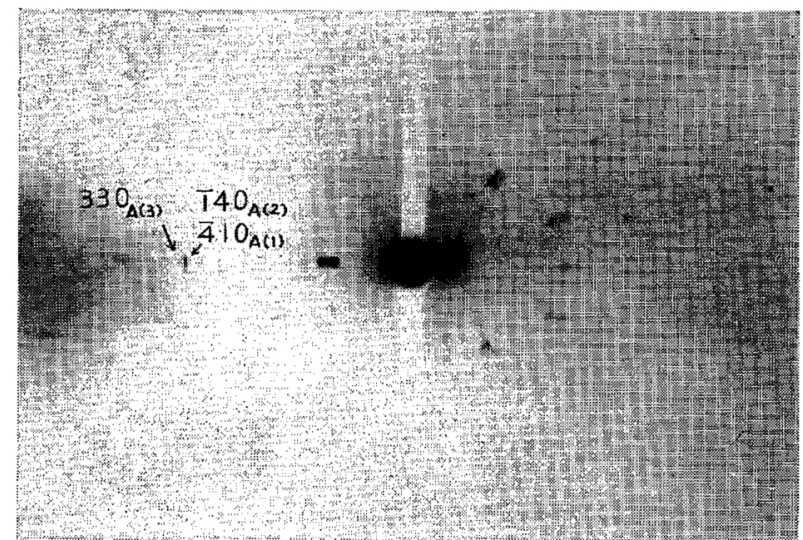

(c)

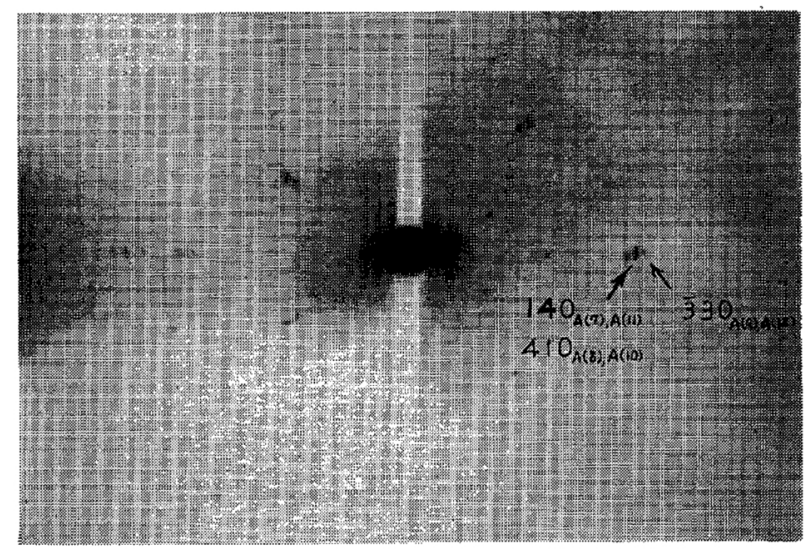

(d)

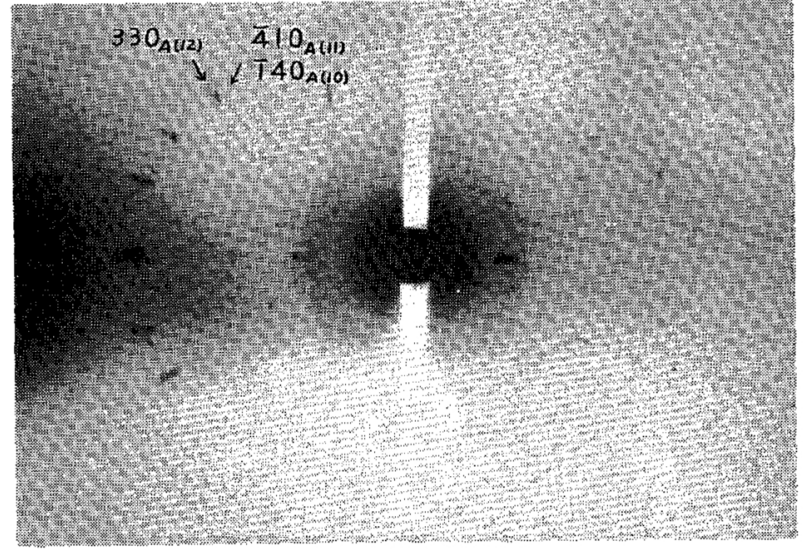

(e)

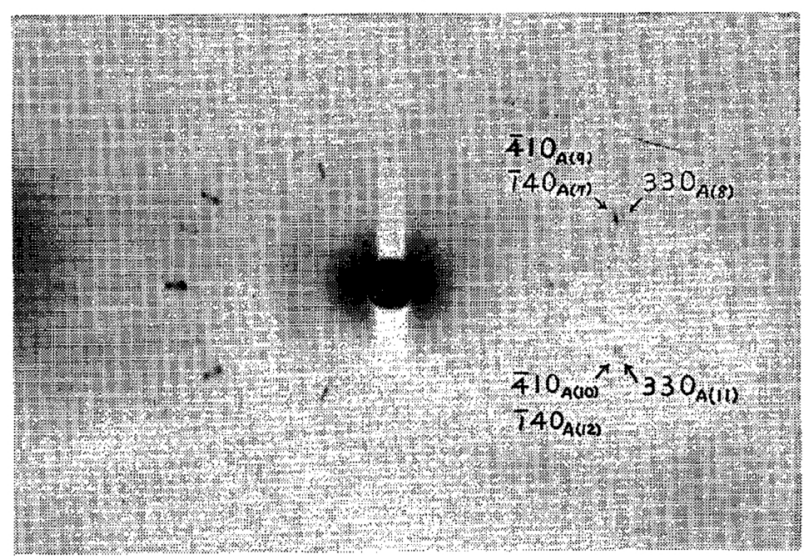

(f)

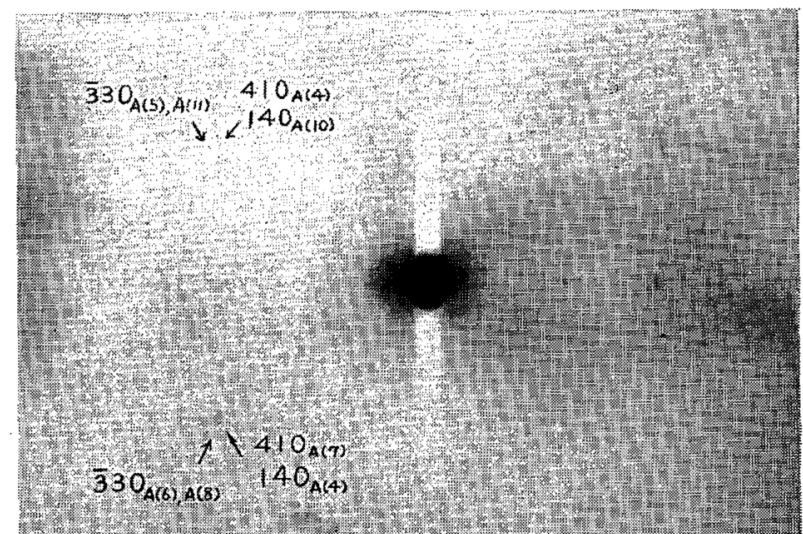

$(\mathrm{g})$

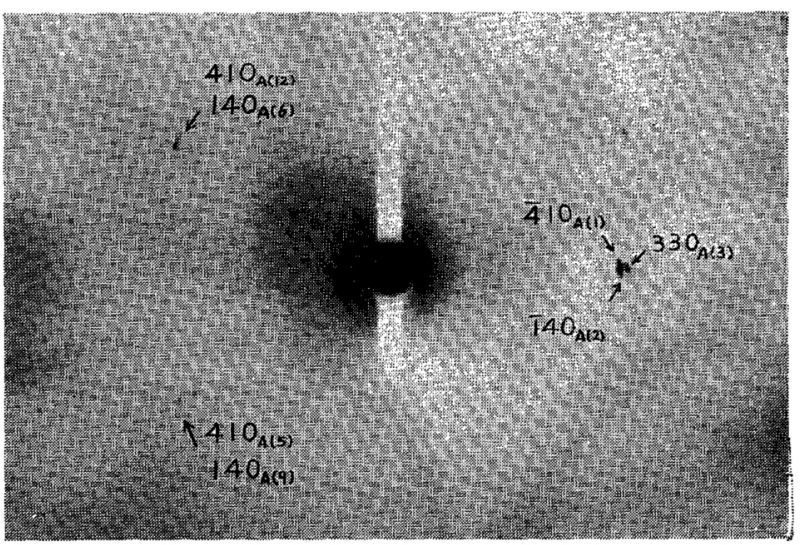

(h)

Photo. 6 Oscillation photographs about $\gamma[0 \overline{1} 1]$ axis, $\mathrm{Cr}-K \alpha$ radiation. Specimen : $815^{\circ} \mathrm{C}, 218 \mathrm{hrs.}$; camera diameter $70 \mathrm{~mm}$.
(a) $\Theta=8.3^{\circ} \sim 10.3^{\circ}$
(b) $\Theta=15.3^{\circ} \sim 16.7^{\circ}$
(c) $\Theta=22.2^{\circ} \sim 24.5^{\circ}$
(d) $\Theta=31.6^{\circ} \sim 33.8^{\circ}$
(e) $\Theta=47.8^{\circ} \sim 49.8^{\circ}$
(f) $\Theta=58.2^{\circ} \sim 60.3^{\circ}$
(g) $\Theta=84.5^{\circ} \sim 86.5^{\circ}$
(h) $\Theta=87.5^{\circ} \sim 89.5^{\circ}$ 
arrows in Fig. 8. The third layer, that is nothing but the basal plane $\left(A^{\prime}\right)$, can be obtained by moving all atoms in $C$ layer $(r(111))$ together by an amount of $a_{0} / \sqrt{ } \overline{6}$ in any one direction of $\gamma[\overline{2} 11], \gamma[1 \overline{2} 1]$ and $\gamma[11 \overline{2}]$. In this case the amount and direction of displacement of individual atom correspond to those of the Burgers vector of a Shockley partial dislocation, whose Burgers vector lies in $\gamma$ (111) plane. In order for the sigma structure unit cell DEFG in Fig. 8 to be completed, the atoms located at the centers of hexagons on successive $\sigma(001)$ planes $A^{\prime}, B^{\prime}, A^{\prime}$ must be shifted by $-c_{0} / 4$.

The consideration given above leads to a suggestion that the sigma nucleation will be easier to occur at lattice defects such as stacking faults and twins. Once the sigma phase is nucleated, its growth will be accomplished by diffusion, for the composition of the sigma precipitate $(43 \% \mathrm{Cr}, 6 \% \mathrm{Ni}$, and iron the remainder $\left.{ }^{(15)}\right)$ is quite different from the initial composition of the alloy used.

Actually both stacking faults and deformation twins were observed in cold-rolled specimens of this alloy, by electron microscopy ${ }^{(16)}$, and the sigma phase precipitation was found to occur preferentially on such lattice defects when they were annealed at sigma forming temperature. These findings together with the result obtained by Guarnieri et al.(12), that the sigma formation is accelerated by cold work, seem to be in accordance with the result of the considerations given above.

(15) M. E. Nicholson, C. H. Samans and F. J. Shortsleeve : Trans. ASM, 44 (1952), 601.

(16) M. Tagaya, S. Nenno and Z. Nishiyama: Unpublished. 